\title{
Perturbative formalism of Lovelock gravity
}

\author{
G. A. Mena Marugán \\ Instituto de Optica "Daza de Valdés", Consejo Superior de Investigaciones Científicas, Serrano 121, 28006 Madrid, Spain
}

(Received 25 February 1992)

\begin{abstract}
In this work we develop a perturbative formalism for the treatment of Lovelock theories of gravity. We consider in detail $D$-dimensional models in Lovelock gravity with an induced metric given by the product of the metrics of two maximally symmetric spaces. We first explicitly obtain the Lovelock action, Hamiltonian constraint, gravitational momenta, and dynamical equations for this type of minisuperspace model. We then apply the perturbative formalism and show how it solves the partial degeneration and multivaluedness problems in the analyzed Lovelock models. We also study the implementation of the proposed perturbative formalism in models whose induced metric is a product of metrics of an arbitrary number of maximally symmetric spaces. We finally comment on the generalization of this formalism to Lovelock gravity in superspace.
\end{abstract}

PACS number(s): 04.50.+h, 04.60.+n

\section{INTRODUCTION}

Modern unification theories of all physical interactions have led to the idea that our Universe possibly possesses more than four dimensions [1]. Therefore, it seems necessary to develop a formalism of multidimensional cosmology. Lovelock theories of gravity [2] can be an appealing candidate for the study of gravitational dynamics in multidimensional manifolds.

There exist several reasons to consider Lovelock theories as a natural generalization of Einstein gravity in spacetimes of more than four dimensions. Lovelock gravitational equations depend on the induced metric only up to its second spacetime derivatives [2], a property which is shared by the general relativity equations. On the other hand, the gravitational Lovelock propagator coincides with the Einsteinian propagator [3]. As a consequence, Lovelock gravity linearized around Minkowski space is ghost-free, and the number of physical degrees of freedom in Lovelock and Einstein gravities turns out to be the same [3-5]. Finally, the Lovelock Lagrangian is formed by a linear combination of dimensionally continued Euler forms, while the Hilbert-Einstein Lagrangian is given by the dimensional continuation of the Euler form of degree one $[3,6]$. Therefore, Lovelock gravity can be considered as a multidimensional topological generalization of Einstein theory of gravity.

Moreover, it has been checked that Lovelock gravity may correspond to the low-energy limit of stringy gravity, at least for some string models [7].

In spite of all these desirable features, Lovelock theories present a series of disadvantages which make their immediate treatment seriously difficult [8-10]. The main disadvantage is the almost nondegenerate character of this type of theory; i.e., there exist gravitational configurations around which the inversion of the relations between the gravitational momenta and the time derivatives of the metric is not possible [11]. Then, one cannot single-valuedly invert these relations in all the configura- tion space, and the Hamiltonian formalism of Lovelock theories is therefore not well defined $[8,9]$.

It has been recently proposed that a perturbative treatment of Lovelock gravity could provide a way out of this problem [11-13]. In this sense, we will assume that the gravitational Lovelock action is an effective action in the low-energy limit, in which the dominant term, the Hilbert-Einstein action, is corrected with higher-order contributions in the Riemann tensor. This interpretation is justified inasmuch as Einstein theory acceptably describes the gravitational dynamics at low energies and curvatures. With this interpretation at hand $[11,12]$, we shall show in this paper that it is possible to develop a perturbative formalism of Lovelock gravity which solves its partial degeneration problems.

Throughout this work, we suppose a gravitational action of the Lovelock form

$$
\begin{aligned}
S=\int_{\mathcal{M}} \sum_{m=1}^{M} & \frac{\bar{L}_{m}}{D-2 m} \epsilon_{a_{1} \cdots a_{D}} R^{a_{1} a_{2}} \cdots R^{a_{2 m-1} a_{2 m}} \\
& \times e^{a_{2 m+1}} \cdots e^{a_{D}}
\end{aligned}
$$

where $\epsilon_{a_{1} \cdots a_{D}}$ is the $D$-dimensional Levi-Civita tensor and $\mathcal{M}$ is the considered manifold of dimension $D$, with a $D$ tetrad $\left\{e^{a}\right\}(a=1, \ldots, D)$ and curvature two-form $R^{a b}$ [14]. The Lovelock coefficients $\bar{L}_{m}$ are real constants, and $M$ is related to the number of dimensions by

$$
M=\operatorname{int}\left(\frac{D-1}{2}\right)
$$

with $\operatorname{int}(x)$ the integer part of $x$.

In order for the linear term in curvature in (1.1) to correspond to the Hilbert-Einstein action, we must have $\bar{L}_{1}=\left[(D-3) ! 16 \pi G_{D}\right]^{-1}, G_{D}$ being the gravitational constant in $D$ dimensions (which must not be confused 
with the effective four-dimensional gravitational constant [15]). We suppose that the Hilbert-Einstein coefficient $L_{1}$ is strictly positive, so that the corresponding theory of gravity turns out to be attractive in the Einsteinian limit.

On the other hand, the Lovelock action (1.1) must be modified with additional surface terms when the manifold $\mathcal{M}$ has a boundary $[16,17]$. We will return to this point later on.

In the following, we will adopt the sign convention of Misner, Thorne, and Wheeler [18] for the Riemann tensor and its contractions. In addition, accepting an ArnowittDeser-Misner (ADM) decomposition for the metric [18], we will keep the value 1 for the time index; the remaining indices will be denoted by lower-case letters $i, j, \ldots$, running from 2 to $D$, unless otherwise stated. For the extrinsic curvature, our convention will be that of Wald [19]: i.e.,

$$
K_{i j}=\frac{1}{2 N}\left[\dot{h}_{i j}-2 D_{(i} N_{j)}\right],
$$

where $N, N^{i}$, and $h_{i j}$ are, respectively, the lapse function, the shift functions, and the induced metric on the constant time surfaces. In (1.3), $D_{i}$ denotes covariant differentiation with respect to the induced metric, the parentheses denote index symmetrization, and the dot designates a time derivative. Finally, in the rest of this work we will only use dimensionless variables, with numerical values expressed in Planck units.

Calling $h$ the determinant of the induced metric, we can define the gravitational Lagrangian associated with (1.1), $\mathcal{L}$, by means of $S=\int d t d^{D-1} x|h|^{1 / 2} \mathcal{L} . t$ denotes time and $d^{D-1} x$ is the differential element corresponding to the remaining coordinates.

We can then obtain the Hamiltonian constraint in vacuum, $\mathcal{H} \equiv-\frac{\partial \mathcal{L}}{\partial N}=0$, which is equivalent to the purely temporal component of the Lovelock equations [2]:

$$
\mathcal{H} \equiv-\sum_{m=1}^{M} \frac{\bar{L}_{m}}{2^{m}}(D-2 m-1) ! \delta_{j_{1} \cdots j_{2 m}}^{i_{1} \cdots i_{2 m}} R_{i_{1} i_{2}}^{j_{1} j_{2}} \cdots R_{i_{2 m-1} i_{2 m}}^{j_{2 m-1} i_{2 m}}=0
$$

where $\delta_{j_{1} \cdots j_{2 m}}^{i_{1} \cdots i_{2 m}}$ is the generalized Kronecker delta.

Using the Gauss-Codazzi relations [18], the Riemann tensor components $R^{i j}{ }_{k l}$ appearing in (1.4) can be rewritten in terms of the extrinsic curvature and the $(D-1)$ dimensional Riemann tensor of the induced metric, $\bar{R}_{k l}^{i j}$ :

$$
R_{k l}^{i j}=\bar{R}_{k l}^{i j}+K_{k}^{i} K_{l}^{j}-K_{l}^{i} K_{k}^{j} .
$$

The gravitational momenta $\pi^{i j}$ conjugate to the induced metric $h_{i j}$ can be obtained from the expressions [8]

$$
\begin{aligned}
\pi_{j}^{i}=\sum_{m=1}^{M} \bar{L}_{m}(D-2 m-1) ! \sum_{s=0}^{m-1} & \frac{(-4)^{m-s}}{2^{s+1}} \frac{m !(m-s) !}{s !(2 m-2 s) !} \\
& \times \delta_{j j_{1} \cdots j_{2 m-1}}^{i i_{1} \cdots i_{2 m-1}} R_{i_{1} i_{2}}^{j_{1} j_{2}} \cdots R_{i_{2 s-1} j_{2 s}}^{j_{2 s-1} j_{2 s}} K_{i_{2 s+1}}^{j_{2 s+1}} \cdots K^{j_{2 m-1}} i_{i_{2 m-1}}
\end{aligned}
$$

We notice that the gravitational Lagrangian $\mathcal{L}$, the Hamiltonian constraint (1.4), and the momenta (1.6) differ by a factor $|h|^{1 / 2}$ from the tensorial densities used in the literature [8]. The adopted definitions, however, will be more convenient for our subsequent calculations.

It can be also seen [8] that expressions (1.4) and (1.6) are related by

$$
\frac{\partial \mathcal{H}}{\partial K_{l n}}=2 K_{i j} \frac{\partial \pi^{i j}}{\partial K_{l n}} .
$$

In addition, the invariance of the theory under $D-1$ diffeomorphisms on the constant time surfaces imply the momentum constraints in vacuum $\mathcal{H}_{i} \equiv-2 D_{j} \pi_{i}^{j}=0$, where $D_{j}$ denotes again covariant differentiation with respect to the induced metric.

The Lovelock action (1.1) can then be rewritten $[8,20]$ :

$$
S=\int d t d^{D-1} x|h|^{1 / 2}\left[\pi^{i j} \dot{h}_{i j}-N \mathcal{H}-N^{i} \mathcal{H}_{i}\right],
$$

up to surface terms.

In order to get the Hamiltonian form of the Lovelock action, we must insert the functional expression of the time derivatives of the induced metric $\dot{h}_{i j}$ in terms of $h_{i j}$ and $\pi^{i j}$ in (1.8). This functional expression should be obtainable from relations (1.6), which depend on $\dot{h}_{i j}$ through the extrinsic curvature $K_{i j}$, and can be seen as an algebraic system of $\frac{D(D-1)}{2}$ equations for the $\frac{D(D-1)}{2}$ unknowns $\dot{h}_{i j}$, or $K_{i j}$. However, owing to the higherorder Lovelock corrections, this system is not linear in $K_{i j}$, except for the Einsteinian case. As a consequence, the global single-valued inversion of (1.6) in terms of $K_{i j}$ cannot in general be done [8], so that the Hamiltonian formalism is not well defined for Lovelock theories, at least in the conventional way.

The outline of this paper is as follows. In Sec. II we introduce the class of minisuperspace models on which we will mainly concentrate in this work. The induced metric of these models is given by the product of the metrics 
of two maximally symmetric spaces. In Sec. III we explicitly obtain the Hamiltonian constraint, gravitational momenta, and equations of motion for these minisuperspaces. Section IV deals with the generalization of the perturbative formalism of Lovelock gravity proposed in Ref. [11] and its subsequent implementation in the mentioned minisuperspace models. Some specific aspects of this implementation are discussed in Sec. V, where we carry out the perturbative inversion of the Hamiltonian constraint and one of the gravitational momenta in terms of the time derivatives of the induced metric. The introduced perturbative formalism needs to be slightly modified when one of the two maximally symmetric spaces has dimension one. The corresponding modifications are studied in Sec. VI. In Sec. VII we extend the application of the proposed perturbative formalism to models with an induced metric given by the product of a generic number of maximally symmetric spaces. In that section we also comment on the generalization of this perturbative formalism to Lovelock gravity in superspace. The obtained results are summarized in Sec. VIII, where we also include some further discussions. Two Appendices are added. In Appendix A we examine the possible singular behavior of the multivalued derivatives-momenta inversion for models with two maximally symmetric spaces, one of them having dimension three. Finally, in Appendix $B$ we work out some calculations which are useful for the analysis of the perturbative inversions presented in Sec. V.

\section{MINISUPERSPACE MODEL}

In this work we will concentrate on minisuperspace models with the metric

$$
d s^{2}=-N^{2}(t) d t^{2}+a^{2}(t) d \Omega_{u}^{2}+b^{2}(t) d \Omega_{v}^{2} ;
$$

$d \Omega_{u}^{2}$ and $d \Omega_{v}^{2}$ denote the metrics of two maximally symmetric spaces of dimensions $u$ and $v$, respectively, and corresponding scalar curvatures $k_{a}$ and $k_{b}$, which can be equal to $+1,0$ or -1 . $a(t)$ and $b(t)$ are the scale factors of these maximally symmetric spaces, $N(t)$ is the lapse function, and the total dimension of the spacetime is $D=1+u+v$.

The gravitational interaction will be described by the Lovelock action (1.1), with the redefinition

$$
\bar{L}_{m}=\frac{L_{m}}{u ! v !} .
$$

We are mainly interested in studying this type of minisuperspaces for the following reasons: (1) we want to extend the perturbative formalism of Lovelock gravity proposed in Ref. [11] beyond the $D$-dimensional isotropic and homogeneous minisuperspace models analyzed in that reference; (2) the minisuperspace (2.1) can be applied, for $u=3$, to cosmological models in which the extra dimensions could become compactified.

Owing to the relevance of these models, Lovelock minisuperspaces of the form (2.1) have been previously considered by several authors [6,21]. For instance, MüllerHoissen has obtained the dynamical equations of the model (2.1) and some of their classical solutions, keeping only up to cubic terms in curvature in the Lovelock action [22]. Demaret et al. have also taken into account quartic contributions in the Riemann tensor, though they have restricted their study to ten-dimensional spacetimes [23]. Verwimp has dealt with the case $u=3$, constant $b(t)$ and a $(D-4)$-torus as the extra maximally symmetric space [24]. Finally, Deruelle and Fariña-Busto have discussed, in generic $D$-dimensional Lovelock gravity, the following particular cases: (i) constant $b(t)$ and $k_{a}=0$, (ii) constant $b(t)$ and $a(t)$, and (iii) $k_{a}=k_{b}=0$ [25]. We will consider the general model (2.1), without additional restrictions, and find the corresponding Lovelock action, Hamiltonian constraint and Euler-Lagrange equations.

Using the $D$-beins formalism, the minisuperspace (2.1) can be described by means of the $D$-tetrad $\left\{e^{a}\right\}(a=$ $1, \ldots, D)$ :

$e^{1}=N(t) d t, \quad e^{i}=a(t) \tilde{e}^{i}, \quad i=2, \ldots, u+1$,

$e^{\alpha}=b(t) \hat{e}^{\alpha}, \quad \alpha=u+2, \ldots, D$

where $\tilde{e}^{i}$ and $\hat{e}^{\alpha}$ correspond to a $u$-tetrad and a $v$-tetrad of the maximally symmetric spaces of dimension $u$ and $v$, respectively, with associated scalar curvatures $k_{a}$ and $k_{b}$. According to Eqs. (2.3) and (2.4), we adopt the notation $i, j, \ldots$ for indices in the maximally symmetric space of dimension $u(i, j=2, \ldots, u+1), \alpha, \beta, \ldots$ for indices in the $v$-dimensional space $(\alpha, \beta=u+2, \ldots, D)$, and 1 for the time index.

The curvature two-form of the Levi-Civita connection corresponding to (2.3) and (2.4) is given by

$$
\begin{aligned}
& R^{11}=0, \quad R^{1 i}=\frac{1}{a N} \frac{d}{d t}\left(\frac{\dot{a}}{N}\right) e^{1} e^{i} \\
& R^{1 \alpha}=\frac{1}{b N} \frac{d}{d t}\left(\frac{\dot{b}}{N}\right) e^{1} e^{\alpha}, \quad R^{i j}=\left(\frac{k_{a}}{a^{2}}+\frac{\dot{a}^{2}}{a^{2} N^{2}}\right) e^{i} e^{j}
\end{aligned}
$$

$R^{i \alpha}=\frac{\dot{a} \dot{b}}{a b N^{2}} e^{i} e^{\alpha}, \quad R^{\alpha \beta}=\left(\frac{k_{b}}{b^{2}}+\frac{\dot{b}^{2}}{b^{2} N^{2}}\right) e^{\alpha} e^{\beta}$,

the dot denoting differentiation with respect to time.

Substituting (2.2)-(2.7) in action (1.1) and integrating by parts the second derivative terms, we obtain after a lengthy and tedious calculation [26] the following minisuperspace Lovelock action, up to surface terms:

$$
S=-\int d t V_{u} V_{v} N a^{u} b^{v} \sum_{m=1}^{M} L_{m} \sum_{n=p_{0}}^{q_{0}} \sum_{r=0}^{s_{0}} \sum_{w=0}^{m-s_{1}} A_{D m n r w}^{0} \frac{\bar{g}^{r} \bar{h}^{w} x^{n-2 r} y^{2 m-n-2 w}}{2 m-1-2 r-2 w}
$$


where $V_{u}$ and $V_{v}$ are the volumes of the maximally symmetric spaces of dimensions $u$ and $v$, and we have introduced the notation

$$
\begin{aligned}
& \bar{g}=\frac{k_{a}}{a^{2}}, \quad \bar{h}=\frac{k_{b}}{b^{2}}, \quad x=\frac{\dot{a}}{a N} \quad y=\frac{\dot{b}}{b N}, \\
& p_{l}=\max (0,2 m-v-l), \quad q_{l}=\min (2 m-l, u), \quad s_{l}=\operatorname{int}\left(\frac{n+l}{2}\right), \\
& A_{\text {Dmnrw }}^{l}=\left(\begin{array}{c}
D-2 m-1+l \\
u-n
\end{array}\right)\left(\begin{array}{c}
m-l \\
w
\end{array}\right)\left(\begin{array}{c}
m-w-l \\
r
\end{array}\right)\left(\begin{array}{c}
2 m-2 r-2 w-l \\
n-2 r
\end{array}\right),
\end{aligned}
$$

$l$ being a non-negative integer $(l \geq 0), \max ($,$) and \min ($,$) the maximum and minimum of the considered numbers.$

On the other hand, assuming that the time interval runs from $t=t_{0}$ to $t=t_{f}$, the totality of the surface terms coming from the integrations by parts can be set in the compact form [26]

$$
\left.V_{u} V_{v} a^{u} b^{v} \sum_{m=1}^{M} \frac{L_{m} 2 m}{D-2 m} \sum_{n=p_{1}}^{q_{1}} \sum_{r=0}^{s_{0}} \sum_{w=0}^{m-1-s_{0}} A_{D m n r w}^{1} \frac{\bar{g}^{r} \bar{h}^{w} x^{n-2 r} y^{2 m-1-n-2 w}}{2 m-1-2 r-2 w}\right|_{t_{0}} ^{t_{f}}
$$

in which we have employed notation (2.9)-(2.11) again.

When the manifold $\mathcal{M}$ under study possesses a boundary $\partial \mathcal{M}$, the Lovelock action (1.1) must be corrected with appropiate surface terms. Actually, these terms are needed if we want to obtain a well posed variational Lovelock problem with a fixed metric on the boundary $\partial \mathcal{M}[17]$. These boundary terms have also a topological justification, as they are given by a linear combination of dimensionally continued Chern-Simons forms which provide the natural boundary corrections to the dimensionally continued Euler forms appearing in the Lovelock Lagrangian [16].

Let us first define the second fundamental form $\Theta$ related to the extrinsic curvature $K_{b}^{a}$ by means of [14]

$$
\Theta^{a b}=(n \cdot n)\left(n^{b} K_{c}^{a}-n^{a} K_{c}^{b}\right) e^{c},
$$

where $n$ is the unit normal to $\partial \mathcal{M}$ and we have used the $D$-tetrad notation: $\left\{e^{a}\right\}, a=1, \ldots, D$. From the second fundamental form, and the Levi-Civita connection $\omega$ of the manifold $\mathcal{M}$, we can construct the connection [16] $\omega_{s}=$ $\omega-s \Theta$, whose associated curvature two-form will be denoted by $R_{s}$. Then, the boundary corrections to the Lovelock action (1.1) [with $\bar{L}_{m}$ given by (2.2)] adopt the expression

$$
-\int_{\partial \mathcal{M}} \sum_{m=1}^{M} \frac{L_{m} m}{u ! v !(D-2 m)} \int_{0}^{1} d s \epsilon_{a_{1} \cdots a_{D}} \Theta^{a_{1} a_{2}} R_{s}^{a_{3} a_{4}} \cdots R_{s}^{a_{2 m-1} a_{2 m}} e^{a_{2 m+1}} \cdots e^{a_{D}} .
$$

For our minisuperspace model, we have, in particular,

$$
\begin{aligned}
& n^{a}=\delta_{1}^{a}, \quad K_{j}^{i}=x \delta_{j}^{i}, \quad K_{\beta}^{\alpha}=y \delta_{\beta}^{\alpha}, \quad K_{\alpha}^{i}=0, \\
& \Theta^{1 i}=x e^{i}, \quad \Theta^{1 \alpha}=y e^{\alpha}, \quad \Theta^{i j}=\Theta^{\alpha \beta}=\Theta^{11}=0, \\
& R_{s}^{i j}=\left[\bar{g}+x^{2}(s-1)^{2}\right] e^{i} e^{j}, \quad R_{s}^{\alpha \beta}=\left[\bar{h}+y^{2}(s-1)^{2}\right] e^{\alpha} e^{\beta}, \\
& R_{s}^{i \alpha}=x y(s-1)^{2} e^{i} e^{\alpha},
\end{aligned}
$$

$\bar{g}, \bar{h}, x$, and $y$ defined by $(2.9)$

Substituting (2.16)-(2.18) in (2.14) we obtain

$$
\begin{aligned}
-\int_{\partial \mathcal{M}} d \Omega_{u} d \Omega_{v} a^{u} b^{v} \sum_{m=1}^{M} \frac{L_{m} 2 m}{D-2 m}\{ & {\left[y \sum_{n=p_{1}}^{q_{2}} \sum_{r=0}^{s_{0}} \sum_{w=0}^{m-1-s_{1}}\left(\begin{array}{c}
D-2 m \\
u-t
\end{array}\right)\right.} \\
& \left.\left.+x \sum_{n=p_{2}}^{q_{1}-1} \sum_{r=0}^{s_{0}} \sum_{w=0}^{m-1-s_{1}}\left(\begin{array}{c}
D-2 m \\
u-1-t
\end{array}\right)\right] B_{m n r w} \frac{\bar{g}^{r} \bar{h}^{w} x^{n-2 r} y^{2 m-2-n-2 w}}{2 m-1-2 r-2 w}\right\},
\end{aligned}
$$

where we have already performed the $s$ integration, and $d \Omega_{u}$ and $d \Omega_{v}$ correspond to the volume elements, for unit scale factors, of the maximally symmetric spaces of dimension $u$ and $v$, respectively. In (2.19) we have used notation $(2.10)$ and 


$$
B_{m n r w}=\left(\begin{array}{c}
m-1 \\
w
\end{array}\right)\left(\begin{array}{c}
m-1-w \\
r
\end{array}\right)\left(\begin{array}{c}
2 m-2-2 r-2 w \\
n-2 r
\end{array}\right)
$$

According to our previous comments on the variation interval for the time coordinate, we will assume that the boundary $\partial \mathcal{M}$ is formed up by a final constant time surface, with $t=t_{f}$, and by an initial surface of constant time $t=t_{0}$, with opposite orientation.

After easy but tedious manipulations, it can then be shown that (2.19) actually cancels the surface terms (2.12) arising from integration by parts of the second time derivatives contributions in the Lovelock action. The minisuperspace Lovelock action corrected with the boundary terms (2.19) thus depends on the scale factors $a$ and $b$ only up to their first time derivatives, and can be expressed as a pure time integral with no additional boundary terms.

\section{DYNAMICAL EQUATIONS}

Once the Lovelock action of the minisuperspace model has been found, we turn now to the explicit expressions of the gravitational momenta, the Hamiltonian constraint, and the Euler-Lagrange equations.

Assuming that the action of the matter content of the system does not depend on the time derivatives of the scale factors $a$ and $b$, and defining the gravitational Lagrangian $\mathcal{L}$ by means of $S=\int d t V_{u} V_{v} a^{u} b^{v} \mathcal{L}$, let us introduce the gravitational momenta

$$
p_{x}=\frac{\partial \mathcal{L}}{\partial(\dot{a} / a)}, \quad p_{y}=\frac{\partial \mathcal{L}}{\partial(\dot{b} / b)}
$$

The canonical momenta conjugate to the scale factors are related to $p_{x}$ and $p_{y}$ in the following way:

$$
\begin{aligned}
& p_{a}=\frac{\partial\left(V_{u} V_{v} a^{u} b^{v} \mathcal{L}\right)}{\partial \dot{a}}=V_{u} V_{v} a^{u-1} b^{v} p_{x} \\
& p_{b}=V_{u} V_{v} a^{u} b^{v-1} p_{y} .
\end{aligned}
$$

From action (2.8) it is straightforward to obtain then

$$
\begin{aligned}
& p_{x}=-\sum_{m=1}^{M} L_{m} \sum_{n=p_{0}}^{q_{0}} \sum_{r=0}^{s_{0}} \sum_{w=0}^{m-s_{1}} \frac{n-2 r}{2 m-1-2 r-2 w} A_{D m n r w}^{0} \bar{g}^{r} \bar{h}^{w} x^{n-2 r-1} y^{2 m-n-2 w} \\
& p_{y}=-\sum_{m=1}^{M} L_{m} \sum_{n=p_{0}}^{q_{0}} \sum_{r=0}^{s_{0}} \sum_{w=0}^{m-s_{1}} \frac{2 m-n-2 w}{2 m-1-2 r-2 w} A_{D m n r w}^{0} \bar{g}^{r} \bar{h}^{w} x^{n-2 r} y^{2 m-n-2 w-1}
\end{aligned}
$$

We can also get the minisuperspace Hamiltonian constraint varying (2.8) with respect to the lapse function. Introducing the generic action for the matter content of the model, $S_{\text {matt }}=\int d t V_{u} V_{v} a^{u} b^{v} \mathcal{L}_{\text {matt }}$ (in which the cosmological constant, if any, is included), the Hamiltonian constraint $\frac{\partial \mathcal{L}}{\partial N}+\frac{\partial \mathcal{L}_{\text {matt }}}{\partial N}=0$, adopts the form

$$
-\mathcal{H} \equiv \sum_{m=1}^{M} L_{m} \sum_{n=p_{0}}^{q_{0}} \sum_{r=0}^{s_{0}} \sum_{w=0}^{m-s_{1}} A_{D m n r w}^{0} \bar{g}^{r} \bar{h}^{w} x^{n-2 r} y^{2 m-n-2 w}=T_{11},
$$

where $\mathcal{H}=-\frac{\partial \mathcal{L}}{\partial N}$ denotes, as in (1.4), the gravitational part of this Hamiltonian constraint, and we have used $\frac{\partial \mathcal{L}_{\text {matt }}}{\partial N}=-T_{11}, T_{11}$ being the energy component of the matter energy-momentum tensor [expressed in the $D$-tetrad indices corresponding to (2.3) and (2.4)].

Making use of (2.8) and (3.3)-(3.5), it can then be checked that

$$
S=\int d t V_{u} V_{v} a^{u} b^{v}\left[\frac{\dot{a}}{a} p_{x}+\frac{\dot{b}}{b} p_{y}-N \mathcal{H}\right]
$$

so that the gravitational Hamiltonian of the model turns out to be

$$
H=V_{u} V_{v} a^{u} b^{v} \mathcal{H}
$$

where we are assuming an implicit functional relation $x\left(p_{x}, p_{y}\right), y\left(p_{x}, p_{y}\right)$, which inverts the relations (3.3) and (3.4) between the gravitational momenta and the derivatives of the scale factors. The elements of the Jacobian matrix $J$ associated with these relations are given by

$$
\partial_{x} p_{x}=-\sum_{m=1}^{M} L_{m} 2 m \sum_{n=p_{2}}^{q_{0}-2} \sum_{r=0}^{s_{0}} \sum_{w=0}^{m-1-s_{1}}\left(\begin{array}{c}
D-2 m-1 \\
u-2-n
\end{array}\right) B_{m n r w} \bar{g}^{r} \bar{h}^{w} x^{n-2 r} y^{2 m-2-n-2 w}
$$




$$
\begin{aligned}
& \partial_{y} p_{y}=-\sum_{m=1}^{M} L_{m} 2 m \sum_{n=p_{0}}^{q_{2}} \sum_{r=0}^{s_{0}} \sum_{w=0}^{m-1-s_{1}}\left(\begin{array}{c}
D-2 m-1 \\
u-n
\end{array}\right) B_{m n r w} \bar{g}^{r} \bar{h}^{w} x^{n-2 r} y^{2 m-2-n-2 w}, \\
& \partial_{y} p_{x}=-\sum_{m=1}^{M} L_{m} 2 m \sum_{n=p_{1}}^{q_{1}-1} \sum_{r=0}^{s_{0}} \sum_{w=0}^{m-1-s_{1}}\left(\begin{array}{c}
D-2 m-1 \\
u-1-n
\end{array}\right) B_{m n r w} \bar{g}^{r} \bar{h}^{w} x^{n-2 r} y^{2 m-2-n-2 w},
\end{aligned}
$$

with $\partial_{x} p_{y}=\partial_{y} p_{x}$, and $B_{m n r w}$ defined in (2.20).

Our minisuperspace model is then almost nondegenerate, as it corresponds to a model in Lovelock gravity; i.e., there exist gravitational configurations with an associated vanishing Jacobian (see also [25]):

$$
\partial_{x} p_{x} \partial_{y} p_{y}-\left[\partial_{y} p_{x}\right]^{2}=0
$$

At these configurations, the local analytic inversion of (3.3) and (3.4) is not possible.

From the Lovelock action (2.8), we can also derive the Euler-Lagrange equations of the scale factors $a$ and $b$, which determine the dynamical evolution of the minisuperspace gravitational variables. Supposing that the matter Lagrangian $\mathcal{L}_{\text {matt }}$ does not depend on the time derivatives $\dot{a}$ and $\dot{b}$, these Euler-Lagrange equations can be expressed as

$$
\left(\begin{array}{ll}
\partial_{x} p_{x} & \partial_{y} p_{x} \\
\partial_{x} p_{y} & \partial_{y} p_{y}
\end{array}\right)\left(\begin{array}{l}
\ddot{a} /\left(a N^{2}\right) \\
\ddot{b} /\left(b N^{2}\right)
\end{array}\right)=\left(\begin{array}{c}
u \sigma_{a}-B \\
v \sigma_{b}-C
\end{array}\right)
$$

where we have called

$\sigma_{a}=\frac{1}{u a^{u-1} N} \frac{\partial\left(a^{u} \mathcal{L}_{\text {matt }}\right)}{\partial a}, \quad \sigma_{b}=\frac{1}{v b^{v-1} N} \frac{\partial\left(b^{v} \mathcal{L}_{\text {matt }}\right)}{\partial b}$
$B=\frac{x}{a^{u-2}} \frac{\partial\left(a^{u-1} p_{x}\right)}{\partial a}+\frac{y}{b^{v-1}} \frac{\partial\left(b^{v} p_{x}\right)}{\partial b}-\frac{1}{a^{u-1} N} \frac{\partial\left(a^{u} \mathcal{L}\right)}{\partial a}$

$C=\frac{y}{b^{v-2}} \frac{\partial\left(b^{v-1} p_{y}\right)}{\partial b}+\frac{x}{a^{u-1}} \frac{\partial\left(a^{u} p_{y}\right)}{\partial a}-\frac{1}{b^{v-1} N} \frac{\partial\left(b^{v} \mathcal{L}\right)}{\partial b}$

Equations (3.12) are a system of nonlinear secondorder differential equations, although linearly dependent on the second time derivatives of the scale factors. The matrix on the left-hand side of (3.12) coincides with the Jacobian matrix $J$ of the derivatives-momenta relations (3.3) and (3.4), and is given by Eqs. (3.8)-(3.10). Finally, in (3.13)-(3.15), the partial derivatives $\partial_{a}, \partial_{b}$ denote partial differentiation with fixed $\dot{a}$ and $\dot{b}$, and not with $x$ and $y$ kept fixed.

The Euler-Lagrange Eqs. (3.12) are equivalent to the purely spatial components of the Lovelock equations $[22,25] . \sigma_{a}$ and $\sigma_{b}$ play then the role of pressures in the respective maximally symmetric spaces of dimensions $u$ and $v[22,23,27]$, as we will confirm shortly.

On the other hand, it can be seen from Eqs. (2.8), (3.3), and (3.4) that, after a careful calculation [26], $B$ and $C$ can be written in the compact form

$$
\begin{aligned}
& B=\sum_{m=1}^{M} L_{m} \sum_{n=p_{0}}^{q_{0}} \sum_{r=0}^{s_{0}} \sum_{w=0}^{m-s_{1}}(n-u) A_{D m n r w}^{0} \bar{g}^{r} \bar{h}^{w} x^{n-2 r} y^{2 m-n-2 w}, \\
& C=\sum_{m=1}^{M} L_{m} \sum_{n=p_{0}}^{q_{0}} \sum_{r=0}^{s_{0}} \sum_{w=0}^{m-s_{1}}(2 m-n-v) A_{D m n r w}^{0} \bar{g}^{r} \bar{h}^{w} x^{n-2 r} y^{2 m-n-2 w} .
\end{aligned}
$$

Using these expressions and (3.8)-(3.10), it is then easy to prove the identity

$$
\left(-\partial_{x} p_{x} \frac{\ddot{a}}{a N^{2}}-\partial_{y} p_{x} \frac{\ddot{b}}{b N^{2}}-B\right) \frac{\dot{a}}{a}+\left(-\partial_{x} p_{y} \frac{\ddot{a}}{a N^{2}}-\partial_{y} p_{y} \frac{\ddot{b}}{b N^{2}}-C\right) \frac{\dot{b}}{b}=\frac{1}{a^{u} b^{v}} \frac{d}{d t}\left(-a^{u} b^{v} \mathcal{H}\right),
$$

where $\mathcal{H}$ is given by the left-hand side of (3.5). Substituting Eqs. (3.12) and the Hamiltonian constraint (3.5), we immediately deduce the conservation law $[22,23,27]$

$\frac{d}{d t}\left(T_{11}\right)+u \frac{\dot{a}}{a}\left(\sigma_{a}+T_{11}\right)+v \frac{\dot{b}}{b}\left(\sigma_{b}+T_{11}\right)=0$.

As we had asserted, $T_{11}, \sigma_{a}$, and $\sigma_{b}$ can be respectively interpreted as the energy density and the pressures in the spaces of dimension $u$ and $v$. Relation (3.19) states then that, per unit comobile volume, the energy variation equals the sum of the works made by the pressures in each of the maximally symmetric spaces of the model.

Before concluding this section, we want to rewrite $B$, $C$, and the elements of the Jacobian matrix appearing in the left-hand side of (3.12) in a more convenient form for the analysis to follow. 
Let us first define the variables

$$
\begin{aligned}
& g=\frac{k_{a}}{a^{2}}+\frac{\dot{a}^{2}}{a^{2} N^{2}}=\bar{g}+x^{2}, \\
& h=\frac{k_{b}}{b^{2}}+\frac{\dot{b}^{2}}{b^{2} N^{2}}=\bar{h}+y^{2}, \\
& f=\frac{2 \dot{a} \dot{b}}{a b N^{2}}=2 x y,
\end{aligned}
$$

which are functional combinations of $\bar{g}, \bar{h}, x$ and $y$, given by (2.9). Then, it is possible to prove that Eqs. (3.8)(3.10), (3.16), and (3.17) can be expressed as

$$
\partial_{x} p_{x}=\sum_{m=1}^{M} L_{m} \sum_{n=p_{2}}^{q_{0}-2}\left(\begin{array}{c}
D-2 m-1 \\
u-2-n
\end{array}\right) \mathcal{F}_{m n}(g, h, f),
$$

$$
\partial_{y} p_{y}=\sum_{m=1}^{M} L_{m} \sum_{n=p_{0}}^{q_{2}}\left(\begin{array}{c}
D-2 m-1 \\
u-n
\end{array}\right) \mathcal{F}_{m n}(g, h, f),
$$

$$
\partial_{x} p_{y}=\partial_{y} p_{x}=\sum_{m=1}^{M} L_{m} \sum_{n=p_{1}}^{q_{1}-1}\left(\begin{array}{c}
D-2 m-1 \\
u-1-n
\end{array}\right) \mathcal{F}_{m n}(g, h, f)
$$

$$
\begin{aligned}
& B=\sum_{m=1}^{M} L_{m} \sum_{n=p_{0}}^{q_{0}}(n-u) \mathcal{G}_{D m n}(g, h, f), \\
& C=\sum_{m=1}^{M} L_{m} \sum_{n=p_{0}}^{q_{0}}(2 m-n-v) \mathcal{G}_{D m n}(g, h, f),
\end{aligned}
$$

with the definitions

$$
\mathcal{F}_{m n}(g, h, f)=-2 \sum_{r=w_{1}}^{s_{0}} \frac{g^{r} h^{m+r-n-1} f^{n-2 r} m !}{(n-2 r) ! r !(m+r-n-1) !},
$$

$$
\begin{aligned}
& \mathcal{G}_{D m n}(g, h, f)=\left(\begin{array}{c}
D-2 m-1 \\
u-n
\end{array}\right) \\
& \quad \times \sum_{r=w_{0}}^{s_{0}} \frac{g^{r} h^{m+r-n} f^{n-2 r} m !}{(n-2 r) ! r !(m+r-n) !} \\
& w_{l}=\max (0, n-m+l) .
\end{aligned}
$$

In (3.21)-(3.27) we have used again notation (2.10).

In a similar way, the Hamiltonian constraint (3.5) can be written as

$$
-\mathcal{H} \equiv \sum_{m=1}^{M} L_{m} \sum_{n=p_{0}}^{q_{0}} \mathcal{G}_{D m n}(g, h, f)=T_{11}
$$

We point out that all the expressions (3.21)-(3.25) and (3.29), which provide the minisuperspace dynamical equations and Hamiltonian constraint, solely depend on the gravitational variables $g, h$, and $f$. The gravitational momenta $p_{x}$ and $p_{y},(3.3)$ and (3.4), are instead functions of the four variables $\bar{g}, \bar{h}, x$, and $y$.

Finally, it can be checked from (3.21)-(3.28) that the dynamical equations (3.12) and the Hamiltonian constraint (3.29) contain, as particular cases, the equations obtained in Refs. [22-25,27] for the simplified models there studied. Equations (3.12) and (3.29) can be applied to any minisuperspace model of the form (2.1) whose matter action does not depend on the time derivatives of the scale factors $a$ and $b$.

\section{PERTURBATIVE FORMALISM}

In this section we will extend the application of the perturbative formalism of Lovelock gravity [11] to Lovelock models of the type (2.1). Because of their cosmological interest, we will pay special attention to models whose induced metric is given by the product of metrics of two compact spherically symmetric spaces, one of them having dimension three, i.e., $k_{a}=k_{b}=1$ and $u=3$. Nevertheless, our discussion will be presented in a general form. In particular, our analysis will include the cases $k_{a}=0$ or $k_{b}=0$ in a natural way.

The gravitational Hamiltonian of the models under study is given by (3.7) and (3.5), where $x$ and $y$ must be expressed in terms of the gravitational momenta, for which one needs to invert (3.3) and (3.4). Expressions (3.3) and (3.4) are algebraic in all their variables: $\bar{g}, \bar{h}, x, y, p_{x}$, and $p_{y}$, and therefore they define some multivalued global analytic functions $\left(x^{(i)}\left(p_{x}, p_{y}, \bar{g}, \bar{h}\right), y^{(i)}\left(p_{x}, p_{y}, \bar{g}, \bar{h}\right)\right)$, where the index $i$ denotes the different branches of such multivalued functions.

In spite of this multivaluedness, in the region of low spatial curvatures $(\bar{g}$ and $\bar{h})$ and momenta $\left(p_{x}\right.$ and $\left.p_{y}\right)$ it is always possible to determine that inversion branch which corresponds to the perturbed single-valued Einsteinian inversion of the derivatives-momenta relations in the presence of the Lovelock corrections. This branch can be obtained by taking into account that the correspondence between $x=y=0$ and $p_{x}=p_{y}=0$, which is valid in Einstein gravity, is not altered by the higher order in curvature Lovelock contributions. Thus, to select the perturbative inversion in the mentioned region, we choose that local inversion which verifies

$$
\begin{aligned}
& x^{(1)}\left(p_{x}=0, p_{y}=0, \bar{g}=0, \bar{h}=0\right)=0, \\
& y^{(1)}\left(p_{x}=0, p_{y}=0, \bar{g}=0, \bar{h}=0\right)=0 .
\end{aligned}
$$

This condition univocally fixes the desired inversion branch, for, if $|J|$ is the Jacobian associated with the derivatives-momenta relations (3.3) and (3.4),

$$
|J|=\partial_{x} p_{x} \partial_{y} p_{y}-\left(\partial_{x} p_{y}\right)^{2}
$$

it is easy to check from Eqs. (3.8)-(3.10) that 
$|J(x=0, y=0, \bar{g}=0, \bar{h}=0)|$

$$
=-\frac{4}{D-2} L_{1}^{2}\left(\begin{array}{c}
D-2 \\
u
\end{array}\right)\left(\begin{array}{c}
D-2 \\
u-1
\end{array}\right) \neq 0,
$$

with $u \geq 1, v \geq 1$.

The analytic germ [28] determined by (4.1) may be analytically continued from $p_{x}=p_{y}=\bar{g}=\bar{h}=0$. The physically interesting region restricts to gravitational momenta $p_{x}$ and $p_{y}$, both real or purely imaginary, and to non-negative $\bar{g}$ and $\bar{h}$ (i.e., to real scale factors $a^{2}>0$, $b^{2}>0$, so that $\bar{g}>0$ for $k_{a}=1, \bar{g}=0$ for $k_{a}=0, \bar{h}>0$ for $k_{b}=1$ and $\bar{h}=0$ for $\left.k_{b}=0\right)$. The regime is Lorentzian for real $p_{x}$ and $p_{y}$, and Euclidean for $p_{x}$ and $p_{y}$ both imaginary. Therefore, it suffices to extend the selected germ by analytic continuation to the above region. This analytic continuation can always be carried out, except at the singularities of the considered inversion.

At least for the case $u=3$ in Lovelock theories with non-negative coefficients $\left(L_{m} \geq 0 \forall m>1\right.$ and $\left.L_{1}>0\right)$, we show in Appendix A that the only possible singularities in the studied physical region are ordinary algebraic singularities [obviating some fine-tuned Lovelock theories, in which the derivative of the polynomial $P(z)=\sum_{m=1}^{M} L_{m} z^{m}$ presents a triple zero]. At an ordinary algebraic singularity, there always exists the limit of the inversions $x^{(i)}, y^{(i)}$. Moreover, if $\left(p_{x}^{0}, p_{y}^{0}, \bar{g}^{0}, \bar{h}^{0}\right)$ is an ordinary singularity of $\left(x^{(1)}, y^{(1)}\right),\left|J\left(x^{0}, y^{0}, \bar{g}^{0}, \bar{h}^{0}\right)\right|=0$ must be satisfied, with $|J|$ given by $(4.2)$ and $\left(x^{0}, y^{0}\right)$ the finite limit of the perturbative inversion at that singularity. Then, the system turns out to be degenerated at the configuration $\left(x^{0}, y^{0}, \bar{g}^{0}, \bar{h}^{0}\right)$. To proceed to the analytic continuation of $\left(x^{(1)}, y^{(1)}\right)$ beyond the ordinary algebraic singularities, while preserving the single valuedness of the inversion, one should generally introduce cuts in the complex plane of the variables $\left(p_{x}, p_{y}, \bar{g}, \bar{h}\right)$. The chosen cuts must determine a simply connected region around the origin of the complex plane $\left(p_{x}, p_{y}, \bar{g}, \bar{h}\right)$ in which the analytic continuation is well defined and provides a single-valued inversion.

In more general Lovelock models than those considered in Appendix A there may also exist, in the physically interesting region, nonordinary algebraic singularities at which the inversions $x^{(1)}$ and/or $y^{(1)}$ could diverge. If such singularities are algebraic, the introduction of additional cuts might be necessary to maintain the single valuedness in the analytic continuation.

In a similar way, we can try to perturbatively invert the Hamiltonian constraint (3.5) and one of the gravitational momenta expressions (3.3) or (3.4). Let us consider the case in which the inverted momentum is $p_{y}$.

Relations (3.4) and (3.5) are algebraic in the variables $p_{y}, T_{11}, \bar{g}, \bar{h}, x$, and $y$, and so they define some multivalued global analytic functions $\left(x^{(i)}\left(p_{y}, T_{11}, \bar{g}, \bar{h}\right), y^{(i)}\left(p_{y}, T_{11}, \bar{g}, \bar{h}\right)\right)$. From expression (3.3), these multivalued functions have an associated momentum $p_{x}$ given by

$$
\begin{aligned}
p_{x} & =p_{x}^{(i)}\left(p_{y}, T_{11}, \bar{g}, \bar{h}\right) \\
& \equiv p_{x}\left(x^{(i)}\left(p_{y}, T_{11}, \bar{g}, \bar{h}\right), y^{(i)}\left(p_{y}, T_{11}, \bar{g}, \bar{h}\right), \bar{g}, \bar{h}\right) .
\end{aligned}
$$

Before carrying on our discussion, we want to point out that, if $\left(x^{0}, y^{0}, T_{11}^{0}, \bar{g}^{0}, \bar{h}^{0}\right)$ is a solution to the Hamiltonian constraint $(3.5)$, then $\left(-x^{0},-y^{0}, T_{11}^{0}, \bar{g}^{0}, \bar{h}^{0}\right)$ must also be a solution, owing to the invariance of the theory under time reversal. This corresponds to the feature that the Wheeler-DeWitt equation in Einstein gravity turns out to be quadratic in the gravitational momenta. It seems thus impossible that, from a perturbative formalism, we could reach a constraint of the form (4.4), since it would be linear in the momentum $p_{x}$.

In fact, the inversion of relations (3.4) and (3.5) in the Einsteinian case leads to a double-valued function, so that the associated momentum $p_{x}$, given by (4.4), is also double valued (except in some special models, treated in Sec. VI). By combining the two branches of this doublevalued momentum, we recover the Einsteinian Hamiltonian constraint as a function of the gravitational momenta, and, therefore, the Wheeler-DeWitt equation.

Related to our comments above is the fact that, for the isotropic and homogeneous models considered in Ref. [11], the perturbative inversion of the Hamiltonian constraint turns out to be of the form

$$
\left(\frac{\dot{a}}{a N}\right)^{2}=\hat{P}_{1}^{-1}\left(\frac{T_{11}}{\bar{L}_{1}(D-1) !}\right)-\frac{1}{a^{2}}
$$

with $\bar{L}_{1}$ defined as in (1.1), and where $\hat{P}_{1}^{-1}(z)$ is the single-valued inversion of the Lovelock polynomial, $\hat{P}(x)=\sum_{m=1}^{M} \bar{L}_{m} x^{m} / \bar{L}_{1}$, obtained by analytic continuation of the local inversion that vanishes at the origin. In agreement with our previous discussion, inversion (4.5) corresponds to a bivalued function

$$
\left(\frac{\dot{a}}{a N}\right)= \pm\left[\hat{P}_{1}^{-1}\left(\frac{T_{11}}{\bar{L}_{1}(D-1) !}\right)-\frac{1}{a^{2}}\right]^{1 / 2}
$$

We see that, for these isotropic and homogeneous models, it is more convenient to consider the inversion of the Hamiltonian constraint as a function of $\left(\frac{\dot{a}}{a N}\right)^{2}$, rather than as a function of $\frac{\dot{a}}{a N}$, for in the latter case the obtained inversions present ordinary algebraic singularities at those points with $\hat{P}_{1}^{-1}\left(T_{11} /\left[\bar{L}_{1}(D-1) !\right]\right)=a^{-2}$, at which $\frac{\dot{a}}{a N}$ vanishes. The appearance of this type of algebraic singularities is inherited, as we will show, by the models with product spaces (2.1).

Considering again the perturbative inversion of relations (3.4) and (3.5), we will have then to determine the two inversion branches which, in the region of low matter energy $\left(T_{11} \simeq 0\right)$, spatial curvatures $(\bar{g}, \bar{h} \simeq 0)$, and gravitational momentum $p_{y} \simeq 0$, can be interpreted as perturbed Einsteinian inversions. These inversion branches (which we call branches 1 and 2) will be obtained in Sec. $\mathrm{V}$. Defining then their associated momenta $p_{x}^{(i)}$ by means of (4.4) and (3.3), we can get a perturbative constraint of the form

$$
\begin{aligned}
p_{x}^{2}- & p_{x}\left[p_{x}\left(x^{(1)}, y^{(1)}, \bar{g}, \bar{h}\right)+p_{x}\left(x^{(2)}, y^{(2)}, \bar{g}, \bar{h}\right)\right] \\
& +\left[p_{x}\left(x^{(1)}, y^{(1)}, \bar{g}, \bar{h}\right) \cdot p_{x}\left(x^{(2)}, y^{(2)}, \bar{g}, \bar{h}\right)\right]=0,
\end{aligned}
$$


which is quadratic in the momentum $p_{x}$.

We can likewise consider the inversion of expressions (3.3) and (3.5). In this case, we arrive at a perturbative constraint in which the roles of $p_{x}$ and $p_{y}$ have been interchanged with respect to (4.7),

$$
\begin{aligned}
p_{y}^{2}- & p_{y}\left[p_{y}\left(x^{(1)}, y^{(1)}, \bar{g}, \bar{h}\right)+p_{y}\left(x^{(2)}, y^{(2)}, \bar{g}, \bar{h}\right)\right] \\
& +\left[p_{y}\left(x^{(1)}, y^{(1)}, \bar{g}, \bar{h}\right) \cdot p_{y}\left(x^{(2)}, y^{(2)}, \bar{g}, \bar{h}\right)\right]=0 .
\end{aligned}
$$

Notice that now $\left(x^{(i)}, y^{(i)}\right)=\left(x^{(i)}\left(p_{x}, T_{11}, \bar{g}, \bar{h}\right)\right.$, $\left.y^{(i)}\left(p_{x}, T_{11}, \bar{g}, \bar{h}\right)\right)(i=1,2)$ are inversion branches of relations (3.3) and (3.5).

The proposed perturbative formalism permits us then to get a single-valued inversion of the derivativesmomenta relations, an inversion which straightforwardly provides us with a single-valued Hamiltonian for the model. The system must also verify constraints (4.7) and (4.8). Choosing in an adequate form both the definition domains and the cuts needed for the single-valuedness of the different considered inversions, so restricting their corresponding ranges, the introduced perturbative formalism must be self-consistent. The gravitational configurations can then be equivalently described by the gravitational momenta or by the derivatives $x$ and $y$ associated with them by means of the obtained inversions. In addition, given a system configuration and a known matter energy density which corresponds to it, the double-valued inversions of the Hamiltonian constraint and any of the expressions of the gravitational momenta must lead again to the value of the derivatives $x$ and $y$ at that configuration. Thus, once the ranges of the different inversions have been properly restricted, all the analyzed perturbative constraints can be considered as equivalent to the perturbative Hamiltonian constraint.

It can then be checked that all these constraints are first class [29]. When quantizing the system, the quantum operators corresponding to them must therefore cancel the wave functions of the physical states, at least in semiclassical approximation. In particular, the quantization of the perturbative Hamiltonian constraint provides the generalized Wheeler-DeWitt equation for the considered Lovelock models.

\section{INVERSION OF THE HAMILTONIAN CONSTRAINT AND ANY OF THE DERIVATIVES-MOMENTA RELATIONS}

We would like to determine now the two branches of inversion of relations (3.4) and (3.5) that can be interpreted as perturbed Einsteinian inversions in the region of low spatial curvatures and matter energies. The choice of these branches is not as immediate as for the case of the inversion of the derivatives-momenta relations. At least in an intuitive form, the desired branches should lead to vanishing derivatives $x$ and $y$ in the limit $p_{y}=T_{11}=\bar{g}=\bar{h}=0$, since in such a limit the Einsteinian inversions satisfy $x=y=0$ and this correspondence is not altered by the introduction of the higherorder Lovelock corrections. However, these same argu- ments imply that the searched inversion branches must coincide at $p_{y}=T_{11}=\bar{g}=\bar{h}=0$, and that point must then be an ordinary algebraic singularity of the discussed inversions.

Before trying to find the analytic germs for the required branches, it is convenient to derive the expression of the Jacobian matrix $J_{x y}^{T p_{y}}$ associated with the studied relations (3.4) and (3.5).

In our minisuperspace model, the gravitational Hamiltonian is given by (3.7), where $\mathcal{H}$ can be found in (3.5) as a function of $\bar{g}, \bar{h}, x$, and $y$, as defined by (2.9), or in (3.29) as a function of $g, h$, and $f$, as defined by (3.20). Considering then that $\mathcal{H}$ implicitly depends on the gravitational momenta through its dependence on $x$ and $y$, we get, by applying the chain rule,

$$
\partial_{x}(\mathcal{H})=\partial_{x} p_{x} \partial_{p_{x}}(\mathcal{H})+\partial_{x} p_{y} \partial_{p_{y}}(\mathcal{H})
$$

and a similar expression with the roles of $x$ and $y$ interchanged. $\partial_{x} p_{x}, \partial_{y} p_{x}$, and $\partial_{y} p_{y}$ are given by (3.8)-(3.10), and the partial derivative $\partial_{x}$ in the left-hand side of (5.1) must be performed with fixed $a, b$, and $y$. On the other hand, assuming as we do that the matter Lagrangian of the model does not depend on the derivatives of the scale factors, it follows from the Hamiltonian equations of the system that

$$
\partial_{p_{x}}(\mathcal{H})=x, \quad \partial_{p_{y}}(\mathcal{H})=y .
$$

From (5.1) and (5.2), we obtain

$$
\begin{aligned}
& \partial_{x} \mathcal{H}=2 \partial_{g} \mathcal{H} x+2 \partial_{f} \mathcal{H} y=\partial_{x} p_{x} x+\partial_{y} p_{x} y, \\
& \partial_{y} \mathcal{H}=2 \partial_{f} \mathcal{H} x+2 \partial_{h} \mathcal{H} y=\partial_{x} p_{y} x+\partial_{y} p_{y} y .
\end{aligned}
$$

The first equalities in (5.3) and (5.4) are trivial, given the explicit dependence of $\mathcal{H}$ on $x$ and $y$ in terms of their functional combinations $g, h$, and $f$. In this sense, $\partial_{f} \mathcal{H}$ denotes partial differentiation of $\mathcal{H}(g, h, f)$ with respect to $f$, keeping $g$ and $h$ constant, and $\partial_{g} \mathcal{H}$ and $\partial_{h} \mathcal{H}$ admit similar interpretations.

Employing the notation $\mathbf{v} \equiv(x, y), \boldsymbol{\nabla} \equiv\left(\partial_{x}, \partial_{y}\right)$ and making use of the Jacobian matrix $J$ corresponding to the derivatives-momenta relations, Eqs. (5.3) and (5.4) can be reexpressed

$$
\nabla \mathcal{H}=J(\mathbf{v})
$$

The determinant of the matrix $J_{x y}^{T p_{y}}$ associated with relations (3.4)and (3.5) is then

$$
\left|J_{x y}^{T p_{y}}\right|=\left|\left(\begin{array}{c}
\boldsymbol{\nabla} \mathcal{H} \\
\nabla p_{y}
\end{array}\right)\right|=\left|\left(\begin{array}{c}
x \boldsymbol{\nabla} p_{x} \\
\nabla p_{y}
\end{array}\right)\right|=|J| x
$$

where we have used $\partial_{x} p_{y}=\partial_{y} p_{x}$, and $|J|$ is given by (4.2). Equation (5.6) implies that the local analytic inversion of (3.4) and (3.5) is not possible around those configurations for which either $x$ vanishes or the system turns out to be degenerated, $|J|=0$. We notice that, unlike the case of the inversion of the derivatives-momenta relations, there appear now new singular configurations for $x=0$, which were anticipated in Sec. IV by discussing 
the isotropic and homogeneous models considered in Ref. [11].

In particular, if in the perturbative inversion branches $x^{(i)}$ tends to zero in the limit $p_{y}=T_{11}=\bar{g}=\bar{h}=0$, the analytic germs of these branches cannot be determined at such a point, since it must be a singularity of the desired inversions.

Let us see how we can select the searched inversion branches. Take first relation (3.4) with fixed $p_{y}=0$. We can then obtain a local inversion of (3.4) around $x=\bar{g}=$ $\bar{h}=0, y^{(1)}(x, \bar{g}, \bar{h})$, by requiring that

$$
y^{(1)}(x=0, \bar{g}=0, \bar{h}=0)=0 \text {. }
$$

Since $\partial_{y} p_{y}(x=0, y=0, \bar{g}=0, \bar{h}=0) \neq 0$, this condition univocally determines the chosen inversion. Such an inversion will be well defined at least in a certain neighborhood $B_{1}$ of $(x=0, \bar{g}=0, \bar{h}=0)$. Moreover, as expression (3.4), with $p_{y}=0$, is invariant under time reversal $(x, y) \leftrightarrow(-x,-y)$, it is not difficult to prove that the inversion $y^{(1)}(x, \bar{g}, \bar{h})$ is an odd function of $x$ in $B_{1}$.

From relation (3.5), we will have that, for $p_{y}=0$ and $(x, \bar{g}, \bar{h}) \in B_{1}$,

$$
-\hat{\mathcal{H}}(x, \bar{g}, \bar{h}) \equiv-\mathcal{H}\left(x, y^{(1)}(x, \bar{g}, \bar{h}), \bar{g}, \bar{h}\right)=T_{11},
$$

with $\mathcal{H}\left(x, y^{(1)}, \bar{g}, \bar{h}\right)$ the result of substituting the local inversion $y^{(1)}$ in $\mathcal{H}$. It can be checked that $\hat{\mathcal{H}}$ is an even function of $x$, with $(x, \bar{g}, \bar{h}) \in B_{1}$, and therefore we can consider it as a function of $\left(x^{2}, \bar{g}, \bar{h}\right)$ in $B_{2}, B_{2}$ being the image of $B_{1}$ under $x \rightarrow x^{2}$. We can then define a local inversion of (5.8) around $T_{11}=\bar{g}=\bar{h}=0$, $\left(x^{2}\right)^{(1)}\left(T_{11}, \bar{g}, \bar{h}\right)$, univocally determined by the condition

$$
\left(x^{2}\right)^{(1)}\left(T_{11}=0, \bar{g}=0, \bar{h}=0\right)=0 .
$$

This inversion is possible because

$$
\partial_{x^{2}} \hat{\mathcal{H}}\left(x^{2}=0, \bar{g}=0, \bar{h}=0\right)
$$

$$
=\frac{\left|\tilde{J}\left(x^{2}=0, \bar{g}=0, \bar{h}=0\right)\right|}{2 \partial_{y} \tilde{p}_{y}\left(x^{2}=0, \bar{g}=0, \bar{h}=0\right)} \neq 0,
$$

where $\partial_{y} \tilde{p}_{y}\left(x^{2}, \bar{g}, \bar{h}\right) \equiv \partial_{y} p_{y}\left(x, y^{(1)}, \bar{g}, \bar{h}\right)$ and $\left|\tilde{J}\left(x^{2}, \bar{g}, \bar{h}\right)\right|$ $\equiv\left|J\left(x, y^{(1)}, \bar{g}, \bar{h}\right)\right|,|J|$ given by (4.2) and different from zero at $x=y=\bar{g}=\bar{h}=0$, according to (4.3).

The local inversion $\left(x^{2}\right)^{(1)}$ will be well defined at least in a neighborhood $B_{3}$ of the point $T_{11}=\bar{g}=\bar{h}=0$. Let us take now $w^{0} \equiv\left(T_{11}^{0} \neq 0, \bar{g}=0, \bar{h}=0\right) \in B_{3}$, with $T_{11}^{0}$ real. At $w^{0}$, $\left(x^{2}\right)^{(1)}\left(w^{0}\right) \neq 0$, since $\left(x^{2}\right)^{(1)}$ is single valued in $B_{3}$ for fixed $\bar{g}$ and $\bar{h}$, and it vanishes at $T_{11}=\bar{g}=\bar{h}=0$. We thus obtain two solutions, $x^{(1)}=+\sqrt{\left(x^{2}\right)^{(1)}\left(w^{0}\right)}$ and $x^{(2)}=-\sqrt{\left(x^{2}\right)^{(1)}\left(w^{0}\right)}$, which provide us with the following inversion solutions of (3.4) and (3.5) at $z^{0} \equiv\left(p_{y}=0, w^{0}\right)$ :

$$
\begin{aligned}
& \left(x^{(1)}, y^{(1)}\right)\left(z^{0}\right)=\left(+\sqrt{\left(x^{2}\right)^{(1)}\left(w^{0}\right)}, y^{(1)}\left(+\sqrt{\left(x^{2}\right)^{(1)}\left(w^{0}\right)}, \bar{g}=0, \bar{h}=0\right)\right) \\
& \left(x^{(2)}, y^{(2)}\right)\left(z^{0}\right)=\left(-\sqrt{\left(x^{2}\right)^{(1)}\left(w^{0}\right)}, y^{(1)}\left(-\sqrt{\left(x^{2}\right)^{(1)}\left(w^{0}\right)}, \bar{g}=0, \bar{h}=0\right)\right) .
\end{aligned}
$$

Since, obviously, $x^{(i)}\left(z^{0}\right) \neq 0$ and $\mid J\left(x^{(i)}\left(z^{0}\right), y^{(i)}\left(z^{0}\right)\right.$, $\bar{g}=0, \bar{h}=0) \mid \neq 0(i=1,2)$, Eqs. (5.11) and (5.12) univocally determine two different inversion branches of relations (3.4) and (3.5) around $z^{0}$. Therefore, we can construct the analytic germs of the two desired inversion branches by imposing conditions (5.11) and (5.12), and from these germs we can obtain the double-valued inversion of (3.4) and (3.5) by analytic continuation.

Note that Eqs. (5.11) and (5.12) actually select the perturbative inversions, for, if $T_{11}^{0} \rightarrow 0$ (with $p_{y}=$ $\bar{g}=\bar{h}=0)$, both $\left(x^{(1)}, y^{(1)}\right)$ and $\left(x^{(2)}, y^{(2)}\right)$ tend to $(0,0)$ and coincide in that limit. We also point out that, at $z^{0},\left(x^{(1)}, y^{(1)}\right)=\left(-x^{(2)},-y^{(2)}\right)$. This is due to the fact that relations (3.4) and (3.5) are symmetric under the change $\left(x, y, p_{y}\right) \leftrightarrow\left(-x,-y,-p_{y}\right)$, so that if $\left(x^{(1)}\left(p_{y}, T_{11}, \bar{g}, \bar{h}\right), y^{(1)}\left(p_{y}, T_{11}, \bar{g}, \bar{h}\right)\right)$ is a solution to both relations, so is $\left(-x^{(1)}\left(-p_{y}, T_{11}, \bar{g}, \bar{h}\right)\right.$, $\left.-y^{(1)}\left(-p_{y}, T_{11}, \bar{g}, \bar{h}\right)\right)$, and thus $\left(-x^{(1)},-y^{(1)}\right)$ is also an admissible solution for $p_{y}=0$. Equations (5.11) and (5.12) state then that the second inversion branch has been chosen as the time reversal of the first one, and therefore it suffices to analyze only one of them.

The analytic continuation of the obtained germs is al- ways possible, except for the existence of singularities. Once again, the region of physical interest restricts to non-negative $\bar{g}$ and $\bar{h}\left(\bar{g}>0\right.$ for $k_{a}=1, \bar{g}=0$ for $k_{a}=0$, $\bar{h}>0$ for $k_{b}=1$ and $\bar{h}=0$ for $k_{b}=0$ ), real $T_{11}$ (in general positive) and real or imaginary $p_{y}$.

At least for models with $u=3$ and $D \geq 6$ in Lovelock theories with non-negative coefficients, $L_{m} \geq 0 \forall m>1$ and $L_{1}>0$ [and except at the very particular case of theories with associated polynomial $P(z)=\sum_{m=1}^{M} L_{m} z^{m}$ whose derivative presents a triple zero], it can be seen by using arguments as those in Appendix A that the only possible singularities in the region of physical interest are ordinary algebraic singularities. To proceed with the analytic continuation beyond the algebraic singularities (even if they are not ordinary ones, for models or theories other than those commented above), one must introduce, in general, cuts in the complex plane of the variables $\left(p_{y}, T_{11}, \bar{g}, \bar{h}\right)$ in order to conserve the single valuedness of the constructed inversions.

At an ordinary algebraic singularity $\left(p_{y}^{0}, T_{11}^{0}, \bar{g}^{0}, \bar{h}^{0}\right)$ of say $\left(x^{(1)}, y^{(1)}\right)$, the inversion 1 possesses a finite limit $\left(x^{0}, y^{0}\right)$ for which either $x^{0}=0$ or the Jacobian (4.2) vanishes (and thus that singularity also corresponds to a 
singular configuration for the inversion of the derivativesmomenta relations). The appearance of new possible singular configurations (those with $x=0$ ) somewhat complicates the perturbative inversion of relations (3.4) and (3.5).

In Appendix B we show, nevertheless, that if $z^{0} \equiv$ $\left(p_{y}^{0}, T_{11}^{0}, \bar{g}^{0}, \bar{h}^{0}\right)$ is an ordinary algebraic singularity of any of the two perturbative inversion branches, at which it is verified that (1) the two considered branches coincide, with $\left(x^{(1)}, y^{(1)}\right)\left(z^{0}\right)=\left(x^{(2)}, y^{(2)}\right)\left(z^{0}\right)=\left(0, y^{0}\right)$, and (2) $\partial_{y} p_{y}\left(x=0, y^{0}, \bar{g}^{0}, \bar{h}^{0}\right) \neq 0$ and $\left|J\left(x=0, y^{0}, \bar{g}^{0}, \bar{h}^{0}\right)\right| \neq 0$, then the associated constraint (4.7) is analytic at $z^{0}$.

Actually, the two studied branches are the only local inversions of (3.4) and (3.5) around $z^{0}$ whose values at that point are equal to $\left(0, y^{0}\right)$. In addition, the perturbative inversion branches turn out (Appendix B) to be analytic functions around $z^{0}$ of the variables $p_{y}, \bar{g}, \bar{h}$, and $\xi^{1 / 2}$, with

$$
\xi=-T_{11}-\mathcal{H}\left(x=0, y\left(x=0, p_{y}, \bar{g}, \bar{h}\right), \bar{g}, \bar{h}\right),
$$

and $y\left(x, p_{y}, \bar{g}, \bar{h}\right)$ the local inversion of relation (3.4) determined by the condition $y\left(x=0, p_{y}^{0}, \bar{g}^{0}, \bar{h}^{0}\right)=y^{0}$. We notice that, in particular, $\xi\left(z^{0}\right)=0$.

Defining $p_{x}^{(i)}(i=1,2)$ as in (4.4), we also prove in Appendix B that the functional combinations $\left[p_{x}^{(1)}+p_{x}^{(2)}\right]$ and $\left[p_{x}^{(1)} \cdot p_{x}^{(2)}\right]$ are analytic functions of $p_{y}, \bar{g}, \bar{h}$, and $\xi$, or, equivalently, of $p_{y}, T_{11}, \bar{g}$, and $\bar{h}$, around $z^{0}$. Thus, constraint (4.7) is analytic at $z^{0}$.

Moreover, it is not difficult to see (Appendix B) that all the points around $z^{0}$ for which $\xi=0$ are also ordinary algebraic singularities of the inversions $\left(x^{(i)}, y^{(i)}\right)$ $(i=1,2)$, and that both branches of inversion coincide at such points, with $x^{(i)}=0$. This statement also implies that the two perturbative branches of inversion coincide, and constraint (4.7) is analytic, at least at all those ordinary algebraic singularities for which some $x^{(i)}=0$ $(i=1,2)$ connectable with the origin of the complex plane $\left(p_{y}, T_{11}, \bar{g}, \bar{h}\right)$ by a path along which

$$
\begin{array}{r}
\left|J\left(x^{(i)}, y^{(i)}, \bar{g}, \bar{h}\right)\right| \neq 0, \quad \partial_{y} p_{y}\left(x^{(i)}, y^{(i)}, \bar{g}, \bar{h}\right) \neq 0, \\
x^{(i)}=0, \quad
\end{array}
$$

where $\left(x^{(i)}, y^{(i)}\right)(i=1,2)$ are the inversions obtained by analytic continuation from the origin as functions of the variables $p_{y}, \bar{g}, \bar{h}$, and $\xi^{1 / 2}$ along such a path.

In particular, since at $p_{y}=T_{11}=\bar{g}=\bar{h}=0$ we have $\left(x^{(i)}, y^{(i)}\right)=(0,0)$, for both $i=1$ and 2 , and $|J| \neq 0$ and $\partial_{y} p_{y} \neq 0$ around $x=y=\bar{g}=\bar{h}=0$, it is straightforward to see that there always exists a simply connected region around the origin of the complex plane $\left(p_{y}, T_{11}, \bar{g}, \bar{h}\right)$ in which the perturbative constraint (4.7) is fully analytic. In that simply connected region, the cuts that could be needed to construct the single-valued perturbative inversion branches are completely irrelevant with respect to the associated perturbative constraint (4.7).

Let us finally notice that constraint (4.7) is at least continuous at any ordinary algebraic singularity with vanishing $x^{(i)}(i=1$ or 2$)$ for which one of the above assumptions (1) or (2) is not satisfied.

\section{PERTURBATIVE FORMALISM FOR $v=1$ MODELS}

In this section we will examine the peculiarities of the perturbative formalism presented in Sec. IV when it is applied to models of the type (2.1) in which one of the maximally symmetric spaces has dimension one, e.g., $v=$ 1 and $u=D-2$.

In these cases, the perturbative inversion of the derivatives-momenta relations and the inversion of momentum $p_{x}$ and the Hamiltonian constraint can be carried out following the discussion in Secs. IV and V. However, the procedure to obtain the perturbative inversion of momentum $p_{y}$ and the Hamiltonian constraint, given by expressions (3.4) and (3.5), must be modified now, so that constraint (4.7) is no longer valid.

In Einstein gravity, the Hamiltonian constraint for this type of model is linear in $p_{x}$. We then expect that the perturbative constraint corresponding to (4.7) should not be quadratic in $p_{x}$, and it should be replaced by a constraint linearly dependent on this gravitational momentum.

The distinctive feature of the $v=1$ models (2.1) is that the momentum $p_{y}$ is independent of $y$, i.e., $\partial_{y} p_{y}$ vanishes identically, as can be easily checked from Eq. (3.9). Then, the Jacobian matrix $J$ associated with the derivatives-momenta relations turns out to be triangular. Moreover, since from (5.4) it follows that $\partial_{y} p_{y}=2 \partial_{h} \mathcal{H}$, we will have then that $\partial_{h} \mathcal{H}=0$, and the gravitational part of the Hamiltonian constraint must be a function of $g$ and $f$ only. On the other hand, being $\partial_{y} p_{y}=0, \partial_{x} p_{y}$ can solely depend on $\bar{g}, \bar{h}$, and $x$; furthermore, taking into account that $\partial_{x} p_{y}$ is in fact a polynomial of $g, h$, and $f, \partial_{x} p_{y}$ must be a function of the sole variable $g$ :

$$
\partial_{x} p_{y}=\partial_{y} p_{x}=-2 F(g)
$$

with $F$ a certain polynomial.

The Jacobian $|J|$ given by (4.2) is then equal to $|J|=$ $-4 F^{2}(g)$, and therefore is different from zero if and only if $F(g)$ does not vanish. In particular, at $g=0$ we must have $|J| \neq 0$, according to (4.3), and so $F(0) \neq 0$. Defining then

$$
\begin{aligned}
& g_{1}=\min \{g>0 / F(g)=0\}, \\
& g_{2}=\max \{g<0 / F(g)=0\},
\end{aligned}
$$

it can be proved that the inversion $\left(x^{(1)}, y^{(1)}\right)$ of the derivatives-momenta relations, determined at $p_{x}=p_{y}=$ $\bar{g}=\bar{h}=0$ by condition (4.1), can be analytically continued in the region of real $\bar{g}$ and real or imaginary momenta $p_{x}$ and $p_{y}$ as long as it is verified that $g_{1}>\bar{g}+\left(x^{(1)}\right)^{2}>g_{2}$, irrespective of the finite values of $\bar{h}$ and $y^{(1)}$. The single valuedness of the so obtained inversion is then guaranteed if we, in addition, restrict to values of $\bar{g}$ in the interval $g_{1}>\bar{g}>g_{2}$ (otherwise, it could be necessary to introduce cuts in the complex plane of the inversion variables).

On the other hand, it can be shown also by employing similar arguments to those in Appendix A that, for any model of the type (2.1) with $v=1$, neither the inversion of the derivatives-momenta relations nor the inversion of the Hamiltonian constraint and the momentum $p_{x}$ pos- 
sess singularities at which $x^{(i)}$ diverges (with $i$ generally denoting the different branches of the corresponding multivalued inversions), nor singularities at which, though $x^{(i)}$ possessed a finite limit $x^{0}, y^{(i)}$ diverges, except if, at the associated configuration $\left(x^{0}, \bar{g}^{0}\right), F\left(\bar{g}^{0}+\left(x^{0}\right)^{2}\right)$ vanishes. We notice that the last condition can never happen when $g_{1}>\bar{g}+\left(x^{0}\right)^{2}>g_{2}$.

Let us return to the inversion of relations (3.4) and (3.5). We have already seen that, when $v=1, \partial_{h} \mathcal{H}=0$. Moreover, since Eqs. (5.3) and (5.4) imply that $\partial_{x} p_{y}=$ $\partial_{y} p_{x}=2 \partial_{f} \mathcal{H}$, we conclude from (6.1) that, if $v=1$, $\partial_{f} \mathcal{H}=-F(g)$. Using then that the gravitational part of the Hamiltonian constraint $\mathcal{H}$ is a function of the variables $g, h$, and $f$, we arrive at

$$
-\mathcal{H} \equiv F(g) f+G(g)=T_{11},
$$

where $G(g)$ is a certain polynomial. We must have in addition that $G(0)=0$, as the gravitational part of the Hamiltonian constraint vanishes when $g=h=f=0$.

Finally, from Eqs. (6.4) and (5.3), we obtain

$$
\partial_{x} p_{x}=-2 F^{\prime}(g) f-2 G^{\prime}(g),
$$

with the prime denoting differentiation with respect to $g$. Integrating expressions $(6.1),(6.5)$ and $\partial_{y} p_{y}=0$ we get then

$$
\begin{aligned}
& p_{y}=-2 x \int_{0}^{1} d z F\left(g\left(z^{2}\right)\right), \\
& p_{x}=-2 F(g) y-2 x \int_{0}^{1} d z G^{\prime}\left(g\left(z^{2}\right)\right),
\end{aligned}
$$

where $g\left(z^{2}\right)=\bar{g}+x^{2} z^{2}$ and we have used that $p_{y}(x=$ $0)=0$ and $p_{y}(x=y=0)=0$ to fix the corresponding integration constants.

Relation (6.6) can be inverted according to our perturbative formalism, obtaining a single-valued inversion $x^{(1)}\left(p_{y}, \bar{g}\right)$ which corresponds to the analytic continuation of the germ determined at $p_{y}=\bar{g}=0$ by the condition

$$
x^{(1)}\left(p_{y}=0, \bar{g}=0\right)=0 .
$$

Let us call

$$
\hat{I}(x, \bar{g}) \equiv\left\{x \in \mathbb{R} \times \mathbb{I}, \bar{g} \in\left(g_{2}, g_{1}\right) / g_{1}>\bar{g}+x^{2}>g_{2}\right\}
$$

(with II the imaginary axis) and

$$
I\left(p_{y}, \bar{g}\right) \equiv\left\{p_{y}, \bar{g} / p_{y}=p_{y}(x, \bar{g}),(x, \bar{g}) \in \hat{I}(x, \bar{g})\right\}
$$

where $p_{y}(x, \bar{g})$ is given by (6.6). Then, it can be seen that $I\left(p_{y}, \bar{g}\right)$ is simply connected and that the analytic germ of inversion fixed by (6.8) can be analytically continued from $p_{y}=\bar{g}=0$ at least to the whole region $I\left(p_{y}, \bar{g}\right)$. $I\left(p_{y}, \bar{g}\right)$ being simply connected, the result of this analytic continuation is a single-valued inversion $x^{(1)}$ such that the range of $\left(x^{(1)}, \bar{g}\right)$ in $I\left(p_{y}, \bar{g}\right)$ coincides precisely with $\hat{I}(x, \bar{g})$.

In particular, for Lovelock theories with non-negative coefficients, $L_{m} \geq 0 \forall m>1$ and $L_{1}>0$, we will have $g_{1}=\infty$ from Eqs. (3.10), (6.1), and (6.2), and thus

$$
\hat{I}(x, \bar{g}) \equiv\left\{x \in \mathbb{R} \times \mathbb{I}, \bar{g} \in\left(g_{2}, \infty\right) / \bar{g}+x^{2}>g_{2}\right\}
$$

Denoting then $x^{1}(\bar{g})=\sqrt{\bar{g}-g_{2}}$ and $p_{y}^{1}(\bar{g})=$ $\left|p_{y}\left(i x^{1}(\bar{g}), \bar{g}\right)\right|$, we will have now

$$
I\left(p_{y}, \bar{g}\right) \equiv\left\{p_{y} \in \mathbb{R} \bigcup\left(-i p_{y}^{1}(\bar{g}), i p_{y}^{1}(\bar{g})\right), \bar{g} \in\left(g_{2}, \infty\right)\right\}
$$

and so $\left\{p_{y} \in \mathbb{R}, \bar{g} \geq 0\right\} \subset I\left(p_{y}, \bar{g}\right)$.

On the other hand, from relations (6.6) and (6.7) we get

$p_{y} p_{x}=\left[2 F(g) f+4 x^{2} \int_{0}^{1} d z G^{\prime}\left(g\left(z^{2}\right)\right)\right] \int_{0}^{1} d z F\left(g\left(z^{2}\right)\right)$

and making use of the Hamiltonian constraint (6.4) and of the obtained inversion of $(6.6), x^{(1)}\left(p_{y}, \bar{g}\right)$, we conclude that [at least for $\left.\left(p_{y}, \bar{g}\right) \in I\left(p_{y}, \bar{g}\right)\right]$ :

$p_{x} p_{y}=\left\{2 T_{11}-2 G\left(\bar{g}+\left[x^{(1)}\left(p_{y}, \bar{g}\right)\right]^{2}\right)+4\left[x^{(1)}\left(p_{y}, \bar{g}\right)\right]^{2} \int_{0}^{1} d z G^{\prime}\left(\bar{g}+\left[x^{(1)}\left(p_{y}, \bar{g}\right)\right]^{2} z^{2}\right)\right\} \int_{0}^{1} d z F\left(\bar{g}+\left[x^{(1)}\left(p_{y}, \bar{g}\right)\right]^{2} z^{2}\right)$

This is just the perturbative constraint we wanted to determine, i.e., a function of $p_{y}, T_{11}$, and $\bar{g}$ which is linearly dependent on the momentum $p_{x}$. In order to obtain this constraint, we only have to invert relation (6.6). The cuts in the complex plane of the variables $\left(p_{y}, \bar{g}\right)$, needed for the construction of a single-valued inversion of (6.6) by analytic continuation from $p_{y}=\bar{g}=0$, are thus exactly the same cuts that are necessary to define constraint (6.14).

\section{PERTURBATIVE FORMALISM FOR MODELS WITH MORE THAN TWO DEGREES OF FREEDOM}

In this section we will extend the application of the perturbative formalism of Lovelock gravity to models with an induced $(D-1)$-dimensional metric given by the product of metrics of $P$ maximally symmetric spaces, each of them with an associated scale factor $a_{l}(t)$, scalar curva- 
ture $k_{l}=1,0$ or -1 and dimension $d_{l}(l=1, \ldots, P)$. Particular cases are the isotropic and homogeneous minisuperspace models $(P=1)[11]$ and the models with two maximally symmetric spaces $(P=2)$ analyzed in this work. Our discussion is also applicable to models with a geometry of Bianchi type I, previously considered in Lovelock cosmology by Deruelle when examining the Kasner-type solutions of these kinds of theories [30].

Unless otherwise stated, in the following we will reserve the indices $l, m, n$, and $q$ to denote positive integers implicitly running from 1 to $P$. In addition, we will assume that the matter content of the system can be described by a Lagrangian which does not depend on the time derivatives of the metric, and choose a gauge in which the shift functions vanish and the lapse function is constant.

Let us then introduce the notation

$$
\begin{aligned}
& \bar{g}_{l}=\frac{k_{l}}{a_{l}^{2}}, \quad x_{l}=\frac{\dot{a}_{l}}{a_{l} N}, \\
& g_{l}=\bar{g}_{l}+x_{l}^{2}, \quad f_{l m}=2 x_{l} x_{m} \quad(l \neq m),
\end{aligned}
$$

and define the gravitational Lagrangian $\mathcal{L}$ from the Lovelock action (corrected with boundary terms, so that it only depends on the metric and its first time derivatives $[16,17]$ ) by means of the relation $S=\int d t V(t) \mathcal{L}$, where $V(t)$ is the total volume of the $t$-constant sections. The gravitational part of the Hamiltonian constraint for the models under consideration, $\mathcal{H}=-\frac{\partial \mathcal{L}}{\partial N}$, can be obtained from expression (1.4) as a function of the spatial components of the Riemann tensor. Supposing $\left\{e^{a}\right\},(a=1, \ldots, D)$ is a $D$-tetrad of the studied $D$ dimensional spacetime, with $e^{1}=N d t$ and such that $\left\{e^{i_{l}} / a_{l}\right\}\left(1+\sum_{j<l} d_{j}<i_{l} \leq 1+\sum_{j \leq l} d_{j}\right)$ is a $d_{l}$-tetrad of its corresponding $l$ th maximally symmetric space, the spatial components of the Riemann tensor are

$$
R^{i_{l} j_{l}}=g_{l} e^{i_{l}} e^{j_{l}}, \quad R^{i_{l} j_{m}}=\frac{1}{2} f_{l m} e^{i_{l}} e^{j_{m}} \quad(l \neq m) .
$$

Notice that all these components vanish when $x_{l}=$ $\bar{g}_{l}=0$.

Likewise, the components of the extrinsic curvature can be written as

$$
K_{j_{l}}^{i_{l}}=x_{l} \delta_{j_{l}}^{i_{l}}, \quad K_{j_{m}}^{i_{l}}=0 \quad(l \neq m),
$$

which vanish if $x_{l}=0$.

Inserting (7.2) in (1.4) we conclude that the gravitational part of the Hamiltonian constraint $\mathcal{H}$ is a polynomial of the variables $g_{l}$ and $f_{l m}$ :

$$
\mathcal{H}=\mathcal{H}\left(g_{l}, f_{l m}\right),
$$

Therefore, the Hamiltonian constraint adopts the expression

$$
\mathcal{H}\left(g_{l}, f_{l m}\right)+T_{11}=0
$$

$T_{11}$ being the energy component of the matter energymomentum tensor (in the above-introduced $D$-tetrad indices). Moreover, since the spatial components of the
Riemann tensor vanish when $g_{l}=f_{l m}=0$, we get from (1.4) that $\mathcal{H}\left(g_{l}=0, f_{l m}=0\right)=0$.

Integration of the generic expression (7.4) leads to $\mathcal{L}=\mathcal{L}\left(x_{l}, \bar{g}_{l}\right)$, and the gravitational Lagrangian is thus a polynomial of all $x_{l}$ and $\bar{g}_{l}$. Defining then $\alpha_{l}=\frac{\dot{a}_{l}}{a_{l}}$ and $p_{x_{l}}=\frac{\partial \mathcal{L}}{\partial \alpha_{l}}$, we conclude that

$$
p_{x_{l}}=p_{x_{l}}\left(x_{m}, \bar{g}_{m}\right)
$$

the gravitational momenta $p_{x_{l}}$ are then polynomials of all $x_{m}$ and $\bar{g}_{m}$.

It can be shown from the above definition of $p_{x_{l}}$ that, unless a factor 2 , these momenta must coincide with the result of substituting the explicit form of the spatial components of the Riemann tensor (7.2) and the extrinsic curvature (7.3) in the general expression (1.6) of the Lovelock momenta. Taking then into account that expressions (7.2) and (7.3) vanish when $x_{m}=\bar{g}_{m}=0$, it follows from Eq. (1.6) that $p_{x_{l}}\left(x_{m}=0, \bar{g}_{m}=0\right)=0$. Moreover, Eq. (1.6) implies that the gravitational momenta $p_{x_{l}}$ are odd in $x_{m}$, in the sense that $p_{x_{l}}\left(x_{m}, \bar{g}_{m}\right)=$ $-p_{x_{l}}\left(-x_{m}, \bar{g}_{m}\right)$.

Relation (1.7) reads now

$$
\partial_{x_{l}} \mathcal{H}=\sum_{n=1}^{P} \partial_{x_{n}} p_{x_{l}} x_{n}
$$

where we have suppressed the mentioned factor 2 and used $\partial_{x_{l}} p_{x_{n}}=\partial_{x_{n}} p_{x_{l}}$. On the other hand, from the generic expression (7.4) we conclude that

$$
\partial_{x_{l}} \mathcal{H}=2 \partial_{g_{l}} \mathcal{H} x_{l}+2 \sum_{n \neq l} \partial_{f_{l n}} \mathcal{H} x_{n}
$$

and therefore

$$
2 \partial_{g_{l}} \mathcal{H}=\partial_{x_{l}} p_{x_{l}}, \quad 2 \partial_{f_{l n}} \mathcal{H}=\partial_{x_{l}} p_{x_{n}}=\partial_{x_{n}} p_{x_{l}} \quad(l \neq n)
$$

must be satisfied. In particular, the $\partial_{x_{l}} p_{x_{n}}$ are polynomials of $g_{m}$ and $f_{m q}$.

As in Sec. IV, we can now invert the derivativesmomenta relations by analytically continuing the germ determined at $p_{x_{m}}=\bar{g}_{m}=0$ by the condition

$$
x_{l}^{(1)}\left(p_{x_{m}}=0, \bar{g}_{m}=0\right)=0 .
$$

This is possible because, since the higher-order Lovelock corrections vanish at $x_{l}=\bar{g}_{l}=0$, the Jacobian matrix

$$
J=\left(\partial_{x_{l}} p_{x_{m}}\right)
$$

must coincide at $x_{l}=\bar{g}_{l}=0$ with the corresponding Einsteinian Jacobian matrix, whose determinant is constant and different from zero. Therefore,

$$
\left|J\left(g_{l}=0, f_{l m}=0\right)\right| \neq 0 .
$$

The analytic germ fixed by condition (7.10) can be then analytically continued in the complex plane of the variables $\left(p_{x_{m}}, \bar{g}_{m}\right)$, except for the existence of singularities at which the inversion $x_{l}^{(1)}$ diverges, or of ordinary algebraic singularities at which the determinant of the 
matrix (7.11) vanishes. Nevertheless, by introducing adequate cuts in the considered complex plane, it is always possible to obtain a single-valued inversion of the derivatives-momenta relations which can be interpreted as the perturbed single-valued Einsteinian inversion in the region of low spatial curvatures $\bar{g}_{m}$ and gravitational momenta $p_{x_{m}}$ according to condition (7.10).

Let us discuss now the inversion of the Hamiltonian constraint (7.5) and $P-1$ of the derivatives-momenta relations, for instance those corresponding to the momenta $p_{x_{r}}$, with $r=2, \ldots, P$. The procedure to perturbatively invert the Hamiltonian constraint and any other set of $P-1$ derivatives-momenta relations is completely parallel.

In what follows, we will implicitly understand that the indices $r$ and $s$ are positive integers running from 2 to $P$.

If any of the momenta $p_{x_{r}}, p_{x_{P}}$ say, were independent of all the variables $x_{r}$, then, by extending the analysis of Sec. VI, we would get a perturbative constraint linearly dependent on $p_{x_{1}}$. However, it is not difficult to see that this is never the case when $P \geq 3$. From Eqs. (7.2) and (1.4), the gravitational part of the Hamiltonian constraint $\mathcal{H}$ depends on all the variables $f_{P t}$ $(t=2, \ldots, P-1 ; P-1 \geq 2)$. Using relations (7.9) we conclude then that $\partial_{x_{t}} p_{x_{P}}$ cannot identically vanish $(t=2, \ldots, P-1)$.

Therefore, there must always exist two perturbative branches of inversion of the Hamiltonian constraint and the momenta $p_{x_{r}}$, branches from which one can obtain a constraint similar to (4.7).

From Eq. (7.8) it follows that the determinant of the Jacobian matrix associated with the considered inversions,

$$
J_{x_{l}}^{T p_{x_{r}}}=\left(\begin{array}{c}
\partial_{x_{l}} \mathcal{H} \\
\partial_{x_{l}} p_{x_{r}}
\end{array}\right),
$$

is $\left|J_{x_{l}}^{T p_{x_{r}}}\right|=|J| x_{1}$, with $|J|$ the determinant of the matrix (7.11). As a consequence, the local analytic inversion of (7.5) and $p_{x_{r}}$ is not possible at those gravitational configurations with $x_{1}=0$ or for which $|J|=0$.

The perturbative inversion of the Hamiltonian constraint and $p_{x_{r}}$ can be performed by extending the analysis of Sec. V. First of all, it can be seen that, at $g_{l}=f_{l m}=0$,

$$
\left|\left(\partial_{x_{r}} p_{x_{s}}\right)\left(g_{l}=0, f_{l m}=0\right)\right| \neq 0
$$

The contribution of the higher-order Lovelock corrections to the Jacobian matrix (7.11) vanishes at $g_{l}=f_{l m}=$ 0 , and so this matrix coincides at that point with the corresponding Jacobian matrix in Einstein gravity, which is known to be constant. If $d_{\bar{r}}$ and $d_{\bar{s}}$ are the dimensions of the respective $\bar{r}$ th and $\bar{s}$ th maximally symmetric spaces of the model, with fixed $\bar{r}, \bar{s} \in\{2, \ldots, P\}$, then it can be checked from the Einsteinian expressions that $d_{\bar{s}} \partial_{x_{\bar{r}}} p_{x_{\bar{r}}}=$ $\left(d_{\bar{r}}-1\right) \partial_{x_{\bar{\tau}}} p_{x_{\bar{s}}}$, and vice versa, $\left(d_{\bar{s}}-1\right) \partial_{x_{\bar{s}}} p_{x_{\bar{r}}}=d_{\bar{r}} \partial_{x_{\bar{s}}} p_{x_{\bar{s}}}$, and so there cannot exist two linearly dependent columns or rows in the matrix $\left(\partial_{x_{r}} p_{x_{s}}\right)$. It follows then that (7.14) is always satisfied.

Equation (7.14) allows us to invert the expressions for $p_{x_{r}}$, with fixed $p_{x_{r}}=0$, around $x_{1}=\bar{g}_{l}=0$, obtaining the local inversion $x_{r}^{(1)}\left(x_{1}, \bar{g}_{l}\right)$ which verifies $x_{r}^{(1)}\left(x_{1}=\right.$ $\left.0, \bar{g}_{l}=0\right)=0$. These inversion functions $x_{r}^{(1)}$ are odd in $x_{1}$. Substituting them in the gravitational part of the Hamiltonian constraint, we obtain an even function of $x_{1}, \hat{\mathcal{H}}\left(x_{1}^{2}, \bar{g}_{l}\right)$, such that

$-\hat{\mathcal{H}}\left(x_{1}^{2}, \bar{g}_{l}\right) \equiv-\mathcal{H}\left[x_{1}, x_{r}^{(1)}\left(x_{1}, \bar{g}_{l}\right), \bar{g}_{l}\right]=T_{11}$.

Using the implicit function theorem, it can be seen then that

$$
\begin{aligned}
\partial_{x_{1}^{2}} \hat{\mathcal{H}}\left(x_{1}^{2}=0,\right. & \left.\bar{g}_{l}=0\right) \\
& =\frac{\left|J\left(g_{l}=0, f_{l m}=0\right)\right|}{2\left|\left(\partial_{x_{r}} p_{x_{s}}\right)\left(g_{l}=0, f_{l m}=0\right)\right|} \neq 0,
\end{aligned}
$$

where we have employed Eqs. (7.12) and (7.14).

We can now invert relation (7.15) around $T_{11}=\bar{g}_{l}=0$ by means of a local inversion $\left(x_{1}^{2}\right)^{(1)}\left(T_{11}, \bar{g}_{l}\right)$ which satisfies the condition $\left(x_{1}^{2}\right)^{(1)}\left(T_{11}=0, \bar{g}_{l}=0\right)=0$. Choosing $T_{11}^{0} \neq 0$, real and close to the origin, so that $\left(x_{1}^{2}\right)^{(1)}$ be well defined around $w^{0} \equiv\left(T_{11}^{0}, \bar{g}_{l}=0\right)$, we immediately get the following inversion solutions to the Hamiltonian constraint and the expressions of $p_{x_{r}}$ at $z^{0} \equiv\left(p_{x_{r}}=0, w^{0}\right)$ :

$$
\begin{aligned}
& \left(x_{1}^{(1)}, x_{r}^{(1)}\right)\left(z^{0}\right) \\
& \quad=\left(\sqrt{\left(x_{1}^{2}\right)^{(1)}\left(w^{0}\right)}, x_{r}^{(1)}\left(\sqrt{\left(x_{1}^{2}\right)^{(1)}\left(w^{0}\right)}, \bar{g}_{l}=0\right)\right),
\end{aligned}
$$

$\left(x_{1}^{(2)}, x_{r}^{(2)}\right)\left(z^{0}\right)=-\left(x_{1}^{(1)}, x_{r}^{(1)}\right)\left(z^{0}\right)$.

These solutions can be used as conditions to fix the analytic germs of the two perturbative inversion branches, in a similar way to that discussed in Sec. V. Such germs can then be analytically continued in the complex plane of the variables $\left(p_{x_{r}}, T_{11}, \bar{g}_{l}\right)$, introducing adequate cuts, if necessary, to guarantee the single valuedness of the so obtained inversions.

Finally, following an analysis parallel to that of Appendix $\mathrm{B}$, it can be shown that if at an ordinary algebraic singularity $z^{0} \equiv\left(p_{x_{r}}^{0}, T_{11}^{0}, \bar{g}_{l}^{0}\right)$ of any of the two perturbative inversion branches of the Hamiltonian constraint and $p_{x_{r}}$ it is verified that (1) the two considered branches coincide, with $\left(x_{1}^{(i)}, x_{r}^{(i)}\right)\left(z^{0}\right)=\left(0, x_{r}^{0}\right), i=1,2$, and (2) $\left|J\left(x_{1}=0, x_{r}^{0}, \bar{g}_{l}^{0}\right)\right| \neq 0$ and $\left|\left(\partial_{x_{r}} p_{x_{s}}\right)\left(x_{1}=0, x_{r}^{0}, \bar{g}_{l}^{0}\right)\right| \neq 0$, then the associated perturbative constraint similar to (4.7) is analytic at $z^{0}$.

All the calculations carried out in Appendix B are extendible to the models here studied with the substitution of $x,(\bar{g}, \bar{h}), y, p_{y}$, and $\partial_{y} p_{y}$ in Appendix B for $x_{1}, \bar{g}_{l}, x_{r}$, $p_{x_{r}}$, and $\left|\left(\partial_{x_{r}} p_{x_{s}}\right)\right|$, respectively. In particular, there exist exactly two local inversions around $z^{0}$ which take the value $\left(x_{1}=0, x_{r}^{0}\right)$ at this point. These inversions must correspond then to the two perturbative branches. Moreover, these local inversions, $\left(x_{l}^{(i)}\right)(i=1,2)$ are analytic functions of the variables $p_{x_{r}}, \bar{g}_{l}$, and $\xi^{1 / 2}$ around $z^{0}$, 
with

$\xi=-T_{11}-\mathcal{H}\left(x_{1}=0, x_{r}\left(x_{1}=0, p_{x_{r}}, \bar{g}_{l}\right), \bar{g}_{l}\right)$,

where $x_{r}\left(x_{1}, p_{x_{r}}, \bar{g}_{l}\right)$ is the local inversion of the momenta $p_{x_{r}}$ which satisfies $x_{r}\left(x_{1}=0, p_{x_{r}}^{0}, \bar{g}_{l}^{0}\right)=x_{r}^{0}$. Substituting $\left(x_{l}^{(i)}\right)(i=1,2)$ in the expression of the momentum $p_{x_{1}}$ we get the functions $p_{x_{1}}^{(i)} \equiv p_{x_{1}}\left(x_{l}^{(i)}, \bar{g}_{l}\right)$, which are such that their functional combinations $\left[p_{x_{1}}^{(1)}+p_{x_{1}}^{(2)}\right]$ and $\left[p_{x_{1}}^{(1)} \cdot p_{x_{1}}^{(2)}\right]$ turn out to be analytic functions of $p_{x_{r}}, \bar{g}_{l}$ and $\xi$ (i.e., of $p_{x_{r}}, \bar{g}_{l}$ and $\left.T_{11}\right)$ at $z^{0}$. Thus, the associated constraint, similar to (4.7), is analytic at $z^{0}$.

In addition, all the points around $z^{0}$ for which $\xi=0$ are also ordinary algebraic singularities of the considered inversions $\left(x_{l}^{(i)}\right)(i=1,2)$. At these points, the inversions $\left(x_{l}^{(i)}\right)$ coincide, with $x_{1}^{(i)}=0(i=1,2)$. As a consequence, the perturbative inversion branches coincide at all the ordinary algebraic singularities around the origin of the complex plane $\left(p_{x_{r}}, T_{11}, \bar{g}_{l}\right)$ for which some $x_{1}^{(i)}=0$ $(i=1,2)$, and the corresponding perturbative constraint is analytic at these points. Therefore, there always exists a simply connected region around the origin of the mentioned complex plane in which the constraint generalizing (4.7) is fully analytic. In such a region, the cuts which could be needed to construct the single-valued perturbative inversion branches are completely irrelevant with respect to the associated perturbative constraint, and, in this sense, their choice leads to no ambiguities in the perturbative formalism.

Basically, we have been able to apply our perturbative formalism to these models with product spaces because of the polynomial dependence of the Hamiltonian constraint and the gravitational momenta on the variables $x_{l}$ and $\bar{g}_{l}$. Other properties of these models that have been used through our discussion are the coincidence, at $x_{l}=\bar{g}_{l}=0$, of the Jacobian matrix (7.11) with its corresponding Einsteinian matrix, the existence of relations (7.7), and the "parity" of the Hamiltonian constraint and momenta $p_{x_{l}}$ with respect to the derivatives $x_{l}$, in the sense that, changing the sign of all the $x_{l}$, the momenta vary their sign, while the Hamiltonian constraint remains unchanged. In addition to all this, we have been able to determine the different perturbative inversions essentially because the gravitational part of the Hamiltonian constraint and the gravitational momenta vanish at $x_{l}=\bar{g}_{l}=0$, both in Einstein and in generic Lovelock gravity.

For the more general formulation in superspace, the gravitational part of the Hamiltonian constraint in Lovelock gravity is given by (1.4), and the gravitational momenta by (1.6). From Eqs. (1.4)-(1.7), it is then straightforward to check that all the properties of the models with product spaces listed above are also satisfied in superspace Lovelock gravity, allowing now the dependence of the gravitational variables on all the spacetime coordinates (not only on time) and considering as the $x_{l}$ variables the components of the extrinsic curvature, $K_{j}^{i}$, and as the $\bar{g}_{l}$ variables the components of the spatial $(D-1)$ dimensional Riemann tensor of the induced metric, $\bar{R}_{k l}^{i j}$. Therefore, the perturbative formalism discussed in this work may be generalized to generic Lovelock gravity in superspace, thus retaining all the degrees of freedom of the theory. This generalization will be carried out elsewhere.

\section{CONCLUSIONS AND FURTHER COMMENTS}

In this work we have developed a perturbative formalism for the treatment of Lovelock gravity which can solve the multivaluedness and partial degeneration problems of these kinds of theories. For this formalism to be applicable, Einstein gravity must properly describe the gravitational interaction in the low-energy limit corresponding to low matter energies, gravitational momenta, and spatial curvatures, the limit in which the higher-order Lovelock corrections should be neglectable.

This perturbative formalism was initially proposed [11] for isotropic and homogeneous Lovelock models. In this paper, we have mainly concentrated on extending the application of this formalism to minisuperspace models with an induced metric given by the product of the metrics of two maximally symmetric spaces.

For such models, we have first obtained the expressions of the Lovelock action, gravitational momenta, Hamiltonian constraint, and dynamical equations. The gravitational momenta turn out to be polynomials of the derivatives of the scale factors $x$ and $y(2.9)$ and of the spatial curvatures $\bar{g}$ and $\bar{h}(2.9)$. Owing to this fact, it is possible to get a single-valued inversion of the derivativesmomenta relations. This inversion is fixed in the region of low momenta $p_{x}$ and $p_{y}$ (3.1) and low spatial curvatures $\bar{g}$ and $\bar{h}$ by imposing that it corresponds to the perturbed single-valued Einsteinian inversion in that region. In general, the construction of this single-valued inversion requires the introduction of cuts in the complex plane of its associated variables $\left(p_{x}, p_{y}, \bar{g}, \bar{h}\right)$. However, we have shown that there always exists a simply connected region around the origin of that plane in which the desired single-valued inversion can be analytically obtained without introducing any cuts.

Using this perturbative single-valued inversion of the derivatives-momenta relations, a single-valued Hamiltonian for the system is straightforwardly attained.

On the other hand, and since the Hamiltonian constraint of the model also turns to be a polynomial of the derivatives $x$ and $y$, the spatial curvatures $\bar{g}$ and $\bar{h}$, and the matter energy density $T_{11}$, one can invert in a similar way the expressions of the Hamiltonian constraint and of one of the gravitational momenta in terms of $x$ and $y$. In general (except for those models with a one-dimensional space), this inversion is double valued in Einstein gravity. In Lovelock gravity, it is possible to construct a double-valued inversion which corresponds to the perturbed Einsteinian inversion in the region of low matter energies $T_{11}$, spatial curvatures $\bar{g}$ and $\bar{h}$, and inverted gravitational momentum $p_{x}$ or $p_{y}$. The substitution of this double-valued inversion in the expression of the gravitational momentum which has not been inverted leads then to a constraint which is quadratic in that momentum.

In order to obtain the wanted double-valued inversion, 
one needs in general to introduce appropriate cuts in the complex plane of the variables corresponding to this inversion. There always exists, however, a simply connected region around the origin of such a plane in which the constraint associated with the considered inversion is fully analytic. In this region, any cut that could be needed in the construction of that inversion turns out to be irrelevant with respect to the mentioned constraint, and, therefore, the choice of those cuts does not imply any kind of ambiguities in the perturbative formalism.

When all the cuts and definition domains of the different discussed inversions (and thus their restricted ranges) are properly chosen, all the analyzed constraints derived from these inversions must be equivalent, at least semiclassically, to the perturvative Hamiltonian constraint that can be obtained from the inversion of the derivatives-momenta relations. Finally, the quantization of that Hamiltonian constraint provides us with the generalized Wheeler-DeWitt equation for the Lovelock models under consideration.

In addition, we have implemented the proposed perturbative formalism in Lovelock minisuperspace models with induced metric given by the product of an arbitrary number of maximally symmetric spaces. We have also commented on the generalization of this formalism to Lovelock gravity in superspace.

Among all the possible consequences and applications of this work, we would like to point out the following. The proposed perturbative formalism of Lovelock gravity permits the study of multidimensional cosmological models of physical interest, in which all except four dimensions could become compactified [31]. One could discuss in this way the role played by the higher-order Lovelock corrections in the considered compactification mechanism, and investigate if such corrections may favor the compactification process.

The perturbative formalism of Lovelock gravity here presented can be also implemented in other different, al- most nondegenerate theories. For this to be plausible, such theories must be interpretable as nondegenerate theories modified by corrective terms. An example could be the case of a nonconformally coupled scalar field in Einstein gravity.

Finally, another appealing proposal in Lovelock quantum cosmology consists in performing the Hamiltonian path integral over suitable complex contours [32] along which the single-valued perturbative inversion of the gravitational momenta (in terms of the time derivatives of the induced metric) always be well defined, without introducing any cut. In such a way, the ambiguities both in the choice of the integration contours and in the introduction of cuts for the mentioned inversion could be substantially reduced or, even, disappear in practice.

\section{ACKNOWLEDGMENTS}

I wish to thank Dr. Pedro González Díaz for his decisive suggestions and friendly collaboration. I am also grateful to Dr. N. Deruelle for her encouraging comments, and to L. J. Garay for his enlighting conversations. I want also to give thanks to J. A. Torresano and to Margarita Marcos for their valuable help.

\section{APPENDIX A: ANALYSIS OF SINGULARITIES IN $u=3$ MODELS}

In this appendix we will analyze the possible singular behavior of the multivalued inversion $\left(x^{(i)}, y^{(i)}\right)(i$ denoting the different existing branches) of the derivativesmomenta relations (3.3) and (3.4). We will restrict our considerations to models of cosmological interest with metric of the form (2.1) in which one of the maximally symmetric spaces has dimension three $(u=3)$. When $u=3$, the expressions (3.3) and (3.4) of the gravitational momenta $p_{x}$ and $p_{y}$ can be rewritten in the form

$$
\begin{aligned}
-\frac{p_{x}}{2}= & 2 x^{2} y \sum_{m=2}^{M} L_{m}\left(\begin{array}{c}
m \\
2
\end{array}\right) h^{m-2}+x \sum_{m=1}^{M_{1}} L_{m}(D-2 m-1) m h^{m-1} \\
& +y \int_{0}^{1} d z\left[2 \bar{g} \sum_{m=2}^{M} L_{m}\left(\begin{array}{c}
m \\
2
\end{array}\right) h^{m-2}\left(z^{2}\right)+\sum_{m=1}^{M_{2}} L_{m}\left(\begin{array}{c}
D-2 m-1 \\
2
\end{array}\right) m h^{m-1}\left(z^{2}\right)\right], \\
-\frac{p_{y}}{2}= & x^{3}\left[\frac{2}{3} \sum_{m=2}^{M} L_{m}\left(\begin{array}{c}
m \\
2
\end{array}\right) h^{m-2}+4 y^{2} \sum_{m=3}^{M} L_{m}\left(\begin{array}{c}
m \\
3
\end{array}\right) h^{m-3}\right] \\
& +2 x^{2} y \sum_{m=2}^{M_{1}} L_{m}(D-2 m-1)\left(\begin{array}{c}
m \\
2
\end{array}\right) h^{m-2}+x\left[2 \bar{g} \sum_{m=2}^{M} L_{m}\left(\begin{array}{c}
m \\
2
\end{array}\right) h^{m-2}+\sum_{m=1}^{M_{2}} L_{m}\left(\begin{array}{c}
D-2 m-1 \\
2
\end{array}\right) m h^{m-1}\right] \\
& +y \int_{0}^{1} d z\left[2 \bar{g} \sum_{m=2}^{M_{1}} L_{m}(D-2 m-1)\left(\begin{array}{c}
m \\
2
\end{array}\right) h^{m-2}\left(z^{2}\right)+\sum_{m=1}^{M_{3}} L_{m}\left(\begin{array}{c}
D-2 m-1 \\
3
\end{array}\right) m h^{m-1}\left(z^{2}\right)\right]
\end{aligned}
$$


where we have used the notation of (1.2), (2.9), and (3.20) and defined

$$
\begin{aligned}
& M_{l}=\operatorname{int}\left(\frac{D-1-l}{2}\right), \quad l=1,2,3, \\
& h\left(z^{2}\right)=\bar{h}+y^{2} z^{2} .
\end{aligned}
$$

We will limit our analysis to the region of real scale factors, $a^{2}>0, b^{2}>0$, for spherically symmetric or flat spaces, i.e., with $\bar{g}>0$ for $k_{a}=1, \bar{g}=0$ for $k_{a}=0, \bar{h}>0$ for $k_{b}=1$, and $\bar{h}=0$ for $k_{b}=0$. Finally, we will only consider Lovelock theories with non-negative coefficients, $L_{m} \geq 0 \forall m>1$ and $L_{1}>0$. This type of theory leads to especially attractive physical behaviors $[11,12]$. For theories in which the positiveness of the Lovelock coefficients is not satisfied, or in the cases $k_{a}=-1$ or $k_{b}=-1$, the following analysis can be carried out in a similar way, although the reached conclusions may vary.

Let $\left(p_{x}^{0}, p_{y}^{0}, \bar{g}^{0}, \bar{h}^{0}\right)$ then be a singularity of the multivalued inversion of (A1) and (A2), $\left(x^{(i)}, y^{(i)}\right)$, with $\bar{g}^{0}=\frac{k_{a}}{\left(a^{0}\right)^{2}}$ and $\bar{h}^{0}=\frac{k_{b}}{\left(b^{0}\right)^{2}}$. We shall show that, if $\left(a^{0}\right)^{2}>0$ and $\left(b^{0}\right)^{2}>0$, it is impossible that $x^{(i)}$ or $y^{(i)}$ diverge (for any $i$ ) when we approach the mentioned singularity by any path or sequence of points.

Let us suppose the opposite, i.e., that $\left(x^{(i)}, y^{(i)}\right)$ diverges (at least for some $i$ ) in any of the following ways: (I) $x^{(i)}$ diverges, but $y^{(i)}$ remains finite; (II) $y^{(i)}$ diverges, but $x^{(i)}$ remains finite; or (III) $x^{(i)}$ and $y^{(i)}$ diverge, and separately discuss the possible divergent behaviors.

Case I. Call $y^{0}$ to the limit of $y^{(i)}$ in the given sequence of points, and divide (A1) by $x^{2}$ and (A2) by $x^{3}$. Taking then into account that $\bar{h}^{0} \geq 0$ and $L_{m} \geq 0 \forall m \geq 1$, we deduce that $x^{(i)}$ may diverge when we approach the singularity only if one of the following conditions is satisfied: (a) $y^{0}=0, L_{2}=0$, and $\bar{h}^{0}=0$; (b) $L_{m}=0 \forall m>1$, or (c) $y^{0} \neq 0$ and $P^{\prime \prime \prime}\left(\bar{h}^{0}+\left(y^{0}\right)^{2}\right)=P^{\prime \prime}\left(\bar{h}^{0}+\left(y^{0}\right)^{2}\right)=0$, with

$$
P(h)=\sum_{m=1}^{M} L_{m} h^{m},
$$

the prime denoting differentiation with respect to $h$.

Suppose first that $k_{b}=0$, so that condition (a) could be satisfied for $\left(b^{0}\right)^{2}>0$. Dividing now (A1) by $x$, we conclude that, if $x^{(i)}$ diverges, $L_{1}$ has to vanish, contravening our assumption $L_{1}>0$. We can also exclude the purely Einsteinian case, in which the regular behavior is well known to be guaranteed. We are thus left only with possibility (c) for Lovelock theories other than Einstein gravity. Let us assume then that $P^{\prime \prime \prime}(h)$ and $P^{\prime \prime}(h)$ have, respectively, a zero of order $q$ and $q+1$ at $h^{0}=\bar{h}^{0}+\left(y^{0}\right)^{2}$ (with $q \geq 1$ ). Dividing again (A2) by $x^{2}$ and (A1) by $x$ we conclude that $\left(\bar{h}+\left(y^{(i)}\right)^{2}-h^{0}\right)^{q} x^{(i)}$ must vanish at the singularity, and also that $P^{\prime}$ must have a zero at $h^{0}$. Therefore, an inversion branch $x^{(i)}$ may diverge at a singularity, keeping $y^{(i)}$ finite, only if the derivative of the polynomial $P(h)$ presents a triple zero, $h^{0}$.

Case II. Let $x^{0}$ be the limit of $x^{(i)}$ in the analyzed sequence of points and define

$$
N=\max \left\{m / L_{m} \neq 0\right\} \text {. }
$$

If $N \neq M[M$ given by (1.2)], divide (A1) and (A2) by $y^{2 N-1}$. Assuming that $y^{(i)}$ diverges at the singularity, we arrive at $L_{N}=0$, contradicting our initial hypothesis. Therefore, $y^{(i)}$ cannot diverge in this case.

If $N=M$ and $D$ is even, divide (A1) by $y^{2 M-2}$ and (A2) by $y^{2 M-3}$. Then, if $y^{(i)}$ diverges, we must have $x^{0}=\bar{g}^{0}=L_{M-1}=0$. Suppose then that $k_{a}=0$, and define

$$
s=\max \left\{m / M>m \geq 1, L_{m} \neq 0\right\}
$$

dividing (A1) by $y^{2 s-1}$, we deduce that $x^{(i)}\left(y^{(i)}\right)^{2 M-2 s-1}$ must remain finite when approaching the singularity. However, this behavior is not compatible with that obtained from dividing (A2) by $y^{2 s-1}$, so that $y^{(i)}$ cannot diverge.

Finally, if $N=M$ and $D$ is odd, divide (A1) and (A2) by $y^{2 M-3}$ and $y^{2 M-4}$, respectively. The only possibility for $y^{(i)}$ to diverge is that $x^{0}=\bar{g}^{0}=L_{M-1}=0$. Supposing then that $k_{a}=0$ and following an analysis similar to that carried out for the case $N=M$ and $D$ even, we deduce again that $y^{(i)}$ cannot diverge at the singularity if $x^{(i)}$ remains finite.

Case III. Let us first assume that $x^{(i)}$ and $y^{(i)}$ diverge when we approach the singularity in such a way that $x^{(i)} / y^{(i)}$ tends to infinity. Defining then $N$ as in (A5) and dividing (A1) by $x^{2} y^{2 N-3}$ we easily check the impossibility of this type of behavior.

Suppose now that $y^{(i)} / x^{(i)}$ tends to infinity at the singularity. If $N \neq M$, we can conclude the inconsistency of this divergent behavior by dividing (A1) by $y^{2 N-1}$. When $y^{(i)} / x^{(i)}$ is assumed to tend to infinity, and $N=M$ with even $D$, it suffices to divide (A1) by $x y^{2 M-2}$ to reach identical conclusions. Finally, for $N=M$, odd $D$, and divergent $y^{(i)} / x^{(i)}$, divide (A1) by $x^{2} y^{2 M-3}$ to show that the supposed behavior at the singularity can never be possible.

We have not considered yet the possibility that $x^{(i)}$ and $y^{(i)}$ diverge when approaching the singularity in such a way that $x^{(i)} / y^{(i)}$ tends to a finite limit $z^{0}$ different from zero. Taking then $N$ as in (A5) and dividing (A1) and (A2) by $y^{2 N-1}$, we get

$$
\begin{aligned}
& \left(z^{0}\right)^{2}(N-1)+z^{0}(D-2 N-1)+\left(\begin{array}{c}
D-2 N-1 \\
2
\end{array}\right) \frac{1}{2 N-1}=0 \\
& \left(z^{0}\right)^{3} \frac{(N-1)}{3}(2 N-3)+\left(z^{0}\right)^{2}(N-1)(D-2 N-1)+z^{0}\left(\begin{array}{c}
D-2 N-1 \\
2
\end{array}\right)+\left(\begin{array}{c}
D-2 N-1 \\
3
\end{array}\right) \frac{1}{2 N-1}=0 .
\end{aligned}
$$


It can be proven that (A7) and (A8) are incompatible for any positive integer $N$, so that we can also affirm that it is impossible that $x^{(i)}$ and $y^{(i)}$ simultaneously diverge while keeping their quotient finite and nonvanishing at the singularity. This ends our demonstration.

\section{APPENDIX B: SINGULARITIES \\ OF THE INVERSION OF THE HAMILTONIAN CONSTRAINT AND MOMENTUM $p_{y}$}

In this appendix we will show that if the perturbative double-valued inversion of momentum $p_{y}$ and the Hamiltonian constraint [relations (3.4) and (3.5)] possesses an algebraic singularity at $z^{0} \equiv\left(p_{y}^{0}, T_{11}^{0}, \bar{g}^{0}, h^{0}\right)$, at which it is satisfied: (1) the two inversion branches coincide with $\left(x^{(1)}, y^{(1)}\right)\left(z^{0}\right)=\left(x^{(2)}, y^{(2)}\right)\left(z^{0}\right)=\left(0, y^{0}\right)$, and (2) $\partial_{y} p_{y}\left(x=0, y^{0}, \bar{g}^{0}, h^{0}\right) \neq 0$ and $\left|J\left(x=0, y^{0}, \bar{g}^{0}, \bar{h}^{0}\right)\right| \neq 0$, then the perturbative constraint (4.7) is analytic at $z^{0}$.

Condition (2) allows us to invert relation (3.4) around $\left(x=0, p_{y}^{0}, \bar{g}^{0}, \bar{h}^{0}\right)$, obtaining a local inversion $y\left(x, p_{y}, \bar{g}, \bar{h}\right)$ that verifies $y\left(x=0, p_{y}^{0}, \bar{g}^{0}, \bar{h}^{0}\right)=y^{0}$. Sub- stituting this inversion in the Hamiltonian constraint (3.5), we get

$-\tilde{\mathcal{H}}\left(x, p_{y}, \bar{g}, \bar{h}\right) \equiv-\mathcal{H}\left(x, y\left(x, p_{y}, \bar{g}, \bar{h}\right), \bar{g}, \bar{h}\right)=T_{11}$.

It can be checked by using the implicit function theorem that

$$
\begin{aligned}
& \partial_{x} \tilde{\mathcal{H}}\left(x=0, \omega^{0}\right)=0, \\
& \partial_{x}^{2} \tilde{\mathcal{H}}\left(x=0, \omega^{0}\right)=\frac{\left|J\left(x=0, y^{0}, \bar{g}^{0}, \bar{h}^{0}\right)\right|}{\partial_{y} p_{y}\left(x=0, y^{0}, \bar{g}^{0}, \bar{h}^{0}\right)} \neq 0,
\end{aligned}
$$

with the compact notation $\omega^{0} \equiv\left(p_{y}^{0}, \bar{g}^{0}, \bar{h}^{0}\right)$ and $|J| \neq 0$, $\partial_{y} p_{y} \neq 0$, according to condition (2). Equation (B2) implies that $x=0$ is a double zero of (B1) at $z^{0}$. It is also possible to see that

$\partial_{p_{y}}^{m_{1}} \partial_{\bar{g}}^{m_{2}} \partial_{\bar{h}}^{m_{3}} \partial_{x} \tilde{\mathcal{H}}\left(x=0, \omega^{0}\right)=0, \quad \forall m_{1}, m_{2}, m_{3} \geq 0$.

Expanding $\tilde{\mathcal{H}}$ in a Taylor series around $\left(x=0, \omega^{0}\right)$, we obtain then

$$
\begin{aligned}
\tilde{\mathcal{H}}= & -T_{11}^{0}+\sum_{m=1}^{\infty} \partial_{\omega}^{m} \tilde{\mathcal{H}}\left(x=0, \omega^{0}\right)\left(\omega-\omega^{0}\right)^{m}+\frac{\partial_{x}^{2} \tilde{\mathcal{H}}}{2}\left(x=0, \omega^{0}\right) x^{2} \\
& +\sum_{m=0}^{\infty} \sum_{n=2}^{\infty}, \frac{\partial_{\omega}^{m} \partial_{x}^{n} \tilde{\mathcal{H}}\left(x=0, \omega^{0}\right)}{n !}\left(\omega-\omega^{0}\right)^{m} x^{n}=-T_{11} .
\end{aligned}
$$

In this expression, the prime means that the term $(n=2, m=0)$ must not be considered in the double summation, and we have employed the symbolic notation

$$
\partial_{\omega}^{m} F\left(\omega-\omega^{0}\right)^{m}=\widehat{\sum_{m_{1}, m_{2}, m_{3}}} \frac{\partial_{p_{y}}^{m_{1}} \partial_{\bar{g}}^{m_{2}} \partial_{\bar{h}}^{m_{3}} F}{m_{1} ! m_{2} ! m_{3} !}\left(p_{y}-p_{y}^{0}\right)^{m_{1}}\left(\bar{g}-\bar{g}^{0}\right)^{m_{2}}\left(\bar{h}-\bar{h}^{0}\right)^{m_{3}},
$$

where the caret denotes restriction to $\sum_{i=1}^{3} m_{i}=m$. The two first terms in the right-hand side (RHS) of Eq. (B4) can be rewritten as

$$
-T_{11}^{0}+\sum_{m=1}^{\infty} \partial_{\omega}^{m} \tilde{\mathcal{H}}\left(x=0, \omega^{0}\right)\left(\omega-\omega^{0}\right)^{m}=\mathcal{H}\left(x=0, y\left(x=0, p_{y}, \bar{g}, \bar{h}\right), \bar{g}, \bar{h}\right),
$$

with $y\left(x, p_{y}, \bar{g}, \bar{h}\right)$ the inversion of (3.4) previously introduced. It can be seen that expression (B6) is well defined around $\left(x=0, \omega^{0}\right)$.

We can obtain the implicit function $x\left(p_{y}, T_{11}, \bar{g}, \bar{h}\right)$, locally defined by Eq. (B4) around $z^{0}$, by substituting in (B4) the power series

$$
x=\sum_{q=1}^{\infty} \sum_{m_{1}, m_{2}, m_{3}=0}^{\infty} a_{q m_{1} m_{2} m_{3}}\left(\xi^{1 / 2}\right)^{q}\left(p_{y}-p_{y}^{0}\right)^{m_{1}}\left(\bar{g}-\bar{g}^{0}\right)^{m_{2}}\left(\bar{h}-\bar{h}^{0}\right)^{m_{3}},
$$

where

$$
\xi=-T_{11}-\mathcal{H}\left(x=0, y\left(x=0, p_{y}, \bar{g}, \bar{h}\right), \bar{g}, \bar{h}\right) .
$$

Note that $x$ given by (B7) and (B8) satisfies $x\left(z^{0}\right)=0$.

Since $x=0$ is a double zero of Eq. (B4) at $z^{0}$ and we have supposed that the two branches of inversion of (3.4) and (3.5) coincide at $z^{0}$, with $x^{(i)}=0(i=1,2)$, there must exist just two possible power series of the form (B7) which are solutions to (B4).

In fact, since $\partial_{x}^{2} \tilde{\mathcal{H}}\left(x=0, \omega^{0}\right) \neq 0$, there are only two admissible choices for the coefficient $a_{1000}$ : 


$$
a_{1000}^{(1)}=+\sqrt{\frac{2}{\partial_{x}^{2} \tilde{\mathcal{H}}\left(x=0, \omega^{0}\right)}}, \quad a_{1000}^{(2)}=-\sqrt{\frac{2}{\partial_{x}^{2} \tilde{\mathcal{H}}\left(x=0, \omega^{0}\right)}} .
$$

Once $a_{1000}$ has been fixed, all other coefficients $a_{k r_{1} r_{2} r_{3}}^{(i)}(i=1,2)$ can be obtained from equation

$$
0=\partial_{x}^{2} \tilde{\mathcal{H}}\left(0, \omega^{0}\right) a_{1000}^{(i)} a_{k r_{1} r_{2} r_{3}}^{(i)}+\sum_{m_{1}=0}^{r_{1}} \sum_{m_{2}=0}^{r_{2}} \sum_{m_{3}=0}^{r_{3}} \sum_{n=2}^{k+1}, \frac{\partial_{p_{y}}^{m_{1}} \partial_{\bar{g}}^{m_{2}} \partial_{\bar{h}}^{m_{3}} \partial_{x}^{n} \tilde{\mathcal{H}}\left(x=0, \omega^{0}\right)}{m_{1} ! m_{2} ! m_{3} ! n !}\left[\widehat{\sum} \prod_{l=1}^{n} a_{q(l) s_{1}(l) s_{2}(l) s_{3}(l)}^{(i)}\right]
$$

where the prime means that the term $\left(n=2, m_{1}=m_{2}=\right.$ $\left.m_{3}=0\right)$ must not be included in the quadruple summation, and $\widehat{\sum}$ denotes now the sum over all products of $n$ coefficients $a_{q(l) s_{1}(l) s_{2}(l) s_{3}(l)}^{(i)}$ such that $\{q(1), \ldots, q(n)\}$ $(q(l) \geq 1)$ and $\left\{s_{j}(1), \ldots, s_{j}(n)\right\}\left(j=1,2,3\right.$ and $s_{j}(l) \geq$ $0)$ are ordered sets of $n$ positive integers satisfying $\sum_{l}^{n} q(l)=k+1, \sum_{l}^{n} s_{j}(l)=r_{j}-m_{j}$. It follows then the existence of just two power series given by (B7).

In addition, we have

$$
a_{k r_{1} r_{2} r_{3}}^{(1)}=(-1)^{k} a_{k r_{1} r_{2} r_{3}}^{(2)} .
$$

To show this relation it suffices to take into account that it holds for $a_{1000}^{(i)}$, noticing that, if (B11) is satisfied for every $a_{q m_{1} m_{2} m_{3}}^{(i)}$ with $q \leq k$ and $m_{j} \leq r_{j}(j=1,2,3)$ other than $a_{k r_{1} r_{2} r_{3}}^{(i)}$, then the second term in the RHS of (B10) has a relative sign $(-1)^{k+1}$ for the two different power series, and $a_{1000}^{(i)}$ in the first term changes sign.

As a consequence, the two corresponding power series (B7) verify

$$
x^{(1)}\left(\xi^{1 / 2}, p_{y}, \bar{g}, \bar{h}\right)=x^{(2)}\left(-\xi^{1 / 2}, p_{y}, \bar{g}, \bar{h}\right) .
$$

Substituting (B12) in the previously obtained inversion of $(3.4), y\left(x, p_{y}, \bar{g}, \bar{h}\right)$, we get

$$
\begin{aligned}
y^{(1)}\left(\xi^{1 / 2}, p_{y}, \bar{g}, \bar{h}\right) & \equiv y\left(x^{(1)}\left(\xi^{1 / 2}, p_{y}, \bar{g}, \bar{h}\right), p_{y}, \bar{g}, \bar{h}\right) \\
& =y^{(2)}\left(-\xi^{1 / 2}, p_{y}, \bar{g}, \bar{h}\right)
\end{aligned}
$$

around $z^{0}$.

From (B12) and (B13) we see that $z^{0}$ is indeed an ordinary algebraic singularity of the inversions $\left(x^{(1)}, y^{(1)}\right)$ and $\left(x^{(2)}, y^{(2)}\right)$ (since, at $z^{0}, \xi=0$ ), and that $z^{0}$ is not an isolated singularity, for there exist neighboring singularities of the same type, determined by the local equation $\xi=0$. Relations (B12) and (B13) also imply that, at all such singularities, the two inversion branches $\left(x^{(1)}, y^{(1)}\right)$ and $\left(x^{(2)}, y^{(2)}\right)$ coincide if they do at $z^{0}$. One can show then that if at an ordinary algebraic singularity $z^{0}$ the two considered inversion branches coincide, with $x^{(1)}=0$, $|J| \neq 0$, and $\partial_{y} p_{y} \neq 0$, then these inversion branches do also coincide at all the ordinary algebraic singularities for which some $x^{(i)}=0(i=1,2)$, connectable with $z^{0}$ by a path in the complex plane $\left(p_{y}, T_{11}, \bar{g}, \bar{h}\right)$ satisfying

$$
\begin{array}{r}
\left|J\left(x^{(i)}, y^{(i)}, \bar{g}, \bar{h}\right)\right| \neq 0, \quad \partial_{y} p_{y}\left(x^{(i)}, y^{(i)}, \bar{g}, \bar{h}\right) \neq 0, \\
x^{(i)}=0, \quad
\end{array}
$$

$\left(x^{(i)}, y^{(i)}\right)(i=1,2)$ being the inversions of (3.4) and (3.5) obtained by analytic continuation from $z^{0}$ as functions of $\xi^{1 / 2}, p_{y}, \bar{g}$, and $\bar{h}$ along such a path. In particular, since the two perturbative inversion branches coincide at $p_{y}=T_{11}=\bar{g}=\bar{h}=0$, with $x^{(1)}=x^{(2)}=0, y^{(1)}=y^{(2)}=0$, and $|J(x=y=\bar{g}=\bar{h}=0)|<0$ and $\partial_{y} p_{y}(x=y=\bar{g}=$ $\bar{h}=0)<0$ [see (4.3) and (3.9)], the two perturbative branches coincide at all the ordinary algebraic singularities with some $x^{(i)}=0(i=1,2)$ connected with the origin by a complex path along which conditions (B14) are satisfied.

On the other hand, inserting (B12) and (B13) in the expression of $p_{x}$, we finally get

$$
\begin{aligned}
p_{x}^{(1)} & \left(\xi^{1 / 2}, p_{y}, \bar{g}, \bar{h}\right) \\
& \equiv p_{x}\left(x^{(1)}\left(\xi^{1 / 2}, p_{y}, \bar{g}, \bar{h}\right), y^{(1)}\left(\xi^{1 / 2}, p_{y}, \bar{g}, \bar{h}\right), \bar{g}, \bar{h}\right) \\
& =p_{x}^{(2)}\left(-\xi^{1 / 2}, p_{y}, \bar{g}, \bar{h}\right),
\end{aligned}
$$

around $z^{0} \equiv\left(p_{y}^{0}, T_{11}^{0}, \bar{g}^{0}, \bar{h}^{0}\right)$. The factors appearing in the constraint (4.7) are then given by

$$
\left[p_{x}^{(1)}\left(\xi^{1 / 2}, p_{y}, \bar{g}, \bar{h}\right)+p_{x}^{(1)}\left(-\xi^{1 / 2}, p_{y}, \bar{g}, \bar{h}\right)\right] \equiv F\left(\xi, p_{y}, \bar{g}, \bar{h}\right),
$$

$\left[p_{x}^{(1)}\left(\xi^{1 / 2}, p_{y}, \bar{g}, \bar{h}\right) \cdot p_{x}^{(1)}\left(-\xi^{1 / 2}, p_{y}, \bar{g}, \bar{h}\right)\right] \equiv G\left(\xi, p_{y}, \bar{g}, \bar{h}\right)$,

with $F$ and $G$ certain functions of $\xi$, rather than $\xi^{1 / 2}$, because expressions (B16) and (B17) are even analytic functions of $\xi^{1 / 2}$, so that their series only contain even powers of this variable. Thus, the ordinary algebraic singularity at $\xi=0$ disappears in the constraint (4.7).

We finally notice that, to carry on with the analytic and single-valued continuation of the perturbative inversions $\left(x^{(i)}, y^{(i)}\right)$ beyond the singularity at $z^{0}$, it is necessary to introduce cuts in the complex plane $\left(p_{y}, T_{11}, \bar{g}, \bar{h}\right)$ which are associated with the square root of $\xi$. When crossing these cuts, we interchange inversions $\left(x^{(1)}, y^{(1)}\right)$ and $\left(x^{(2)}, y^{(2)}\right)$, as stated by (B12) and (B13). The constraint (4.7) is however invariant with respect to this interchange of inversions.
[1] M. B. Green, J. H. Schwarz, and E. Witten, Superstring Theory (Cambridge University Press, Cambridge, England, 1987).

[2] D. Lovelock, Aequationes Math. 4, 127 (1970); J. Math.
Phys. 12, 498 (1971).

[3] B. Zumino, Phys. Rep. 137, 109 (1986).

[4] B. Zwiebach, Phys. Lett. 156B, 315 (1985).

[5] I. L. Buchbinder, I. Yu Karataeva, and S. L. Lyakhovich, 
Class. Quantum Grav. 8, 1113 (1991).

[6] F. Müller-Hoissen, Phys. Lett. 163B, 106 (1985).

[7] M. J. Duff, B. E. W. Nilsson, and C. Pope, Phys. Lett. B 173, 69 (1986).

[8] C. Teitelboim and J. Zanelli, Class. Quantum Grav. 4, L125 (1987).

[9] M. Henneaux, C. Teitelboim, and J. Zanelli, Phys. Rev. A 36, 4417 (1987).

[10] Y. Choquet-Bruhat, J. Math. Phys. 29, 1981 (1988).

[11] G. A. Mena Marugán, Phys. Rev. D 42, 2607 (1990).

[12] G. A. Mena Marugán, Class. Quantum Grav. 8, 935 (1991).

[13] J. Z. Simon, Phys. Rev. D 41, 3720 (1990).

[14] T. Eguchi, P. B. Gilkey, and A. J. Hanson, Phys. Rep. 66, 213 (1980).

[15] F. Müller-Hoissen, Europhys. Lett. 3, 1075 (1987).

[16] R. C. Myers, Phys. Rev. D 36, 392 (1987).

[17] F. Müller-Hoissen, Nucl. Phys. B337, 709 (1990).

[18] C. W. Misner, K. S. Thorne, and J. A. Wheeler, Gravitation (Freeman, San Francisco, 1973).

[19] R. M. Wald, General Relativity (University of Chicago Press, Chicago, 1984).

[20] C. Teitelboim, in General Relativity and Gravitation, edited by A. Held (Plenum, New York, 1980).

[21] N. Deruelle and J. Madore, Mod. Phys. Lett. A 1, 237
(1986); D. Lorenz-Petzold, ibid. 3, 827 (1988).

[22] F. Müller-Hoissen, Class. Quantum Grav. 3, 665 (1986).

[23] J. Demaret, H. Caprese, A. Moussiaux, Ph. Tombal, and D. Papadopoulos, Phys. Rev. D 41, 1163 (1990).

[24] T. Verwimp, Class. Quantum Grav. 6, 1655 (1989).

[25] N. Deruelle and L. Fariña-Busto, Phys. Rev. D 41, 3696 (1990).

[26] G. A. Mena Marugán, Ph.D. thesis (Universidad Complutense de Madrid, Madrid, 1991).

[27] B. C. Paul and S. Mukherjee, Phys. Rev. D 42, 2595 (1990).

[28] L. V. Ahlfors, Complex Analysis (McGraw-Hill, New York, 1979).

[29] P. A. M. Dirac, Lectures on Quantum Mechanics, Belfer Graduate School of Science, Monographs Series (Yeshiva University, New York, 1964).

[30] N. Deruelle, Nucl. Phys. B327, 253 (1989).

[31] G. A. Mena Marugán, following paper, Phys. Rev. D 46, 4340 (1992).

[32] J. J. Halliwell and J. Louko, Phys. Rev. D 39, 2206 (1989); 40, 1868 (1989); 42, 3997 (1990); J. J. Halliwell and J. B. Hartle, ibid. 41, 1815 (1990); 43, 1170 (1991); L. J. Garay, J. J. Halliwell, and G. A. Mena Marugán, ibid. 43, 2572 (1991). 